\title{
Multiple Semiclassical Standing Waves for Fractional Nonlinear Schrödinger Equations
}

\author{
Guoyuan Chen
}

\begin{abstract}
Via a Lyapunov-Schmidt reduction, we obtain multiple semiclassical solutions to a class of fractional nonlinear Schrödinger equations. Precisely, we consider

$$
\varepsilon^{2 s}(-\Delta)^{s} u+u+V(x) u=|u|^{p-1} u, \quad u \in H^{s}\left(\mathbf{R}^{n}\right),
$$

where $0<s<1, n>4-4 s, 1<p<\frac{n+2 s}{n-2 s}$ (if $n>2 s$ ) and $1<p<\infty$ (if $n \leq 2 s$ ), $V(x)$ is a non-negative potential function. If $V$ is a sufficiently smooth bounded function with a non-degenerate compact critical manifold $M$, then, when $\varepsilon$ is sufficiently small, there exist at least $l(M)$ semiclassical solutions, where $l(M)$ is the cup length of $M$.
\end{abstract}

\section{Introduction}

Fractional Schrödinger equations are derived from the path integral over Lévy trajectories. It can be applied, for example, to describe the orbits radius for hydrogen-like atoms. (For more details of physical background, see, for example, [29] and the references therein.)

We study the fractional nonlinear Schrödinger equation of form

$$
i \varepsilon \frac{\partial \psi}{\partial t}=\left(-\varepsilon^{2} \Delta\right)^{s} \psi+V(x) \psi-|\psi|^{p-1} \psi \text { in } \mathbf{R}^{n},
$$

where $\varepsilon$ is a small positive constant which is corresponding to the Planck constant, $(-\Delta)^{s}$, $0<s<1$, is the fractional Laplacian, $V(x)$ is a potential function, and $p>1$.

We shall look for the so-called standing wave solutions which are of form

$$
\psi(x, t)=e^{(i / \varepsilon) E t} v(x),
$$

where $v$ is a real-valued function depending only on $x$ and $E$ is some constant in $\mathbf{R}$. The function $\psi$ solves (1.1) provided the standing wave $v(x)$ satisfies

$$
\left(-\varepsilon^{2} \Delta\right)^{s} v+(V(x)+E) v-|v|^{p-1} v=0 \quad \text { in } \mathbf{R}^{n} .
$$

Key words and phrases. fractional Laplacian, Schrödinger equation, semiclassical solutions, concentration phenomena, Lyapunov-Schmidt reduction. 
In what follows, we assume that $E=1$ and $p$ is subcritical. That is, we will study the following equation:

$$
\varepsilon^{2 s}(-\Delta)^{s} u+u+V(x) u=|u|^{p-1} u, \quad u \in H^{s}\left(\mathbf{R}^{n}\right),
$$

where $0<s<1$, and $1<p<\frac{n+2 s}{n-2 s}$ for $n>2 s$, and, $1<p<\infty$ for $n \leq 2 s$.

In quantum mechanics, when $\varepsilon$ tends to zero, the existence and multiplicity of solutions to (1.3) is of importance. We will find multiple solutions $u_{\varepsilon}$ of (1.3) that concentrate near some point $x_{0} \in \mathbf{R}^{n}$ as $\varepsilon \rightarrow 0$. By this we mean that, for all $x \in \mathbf{R}^{n} \backslash\left\{x_{0}\right\}, u_{\varepsilon}(x) \rightarrow 0$ as $\varepsilon \rightarrow 0$. Such kind of solutions are so-called semiclassical standing waves or spike pattern solutions.

When $s=1$, Equation (1.3) is a classical nonlinear Schrödinger equation and the existence of semiclassical standing wave solutions was established by Floer and Weinstein $[23]$, and then $\mathrm{Oh}[\mathbf{3 2}, \mathbf{3 3}]$. There is a large mount of research on this subject in the past two decades. We refer for example to the (far from complete) list of papers $[\mathbf{3}, \mathbf{4}, \mathbf{5}, \mathbf{6}$, $7,16,9,26,30,18,13,17,27,28,34,36,31]$ and the references therein.

When $s \in(0,1)$, the existence of semiclassical solution to Equations (1.3) was obtained by Dávila, del Pino and Wei [15], Chen and Zheng [12]. Precisely, by a LyapunovSchimdt reduction, [15] proved that if $V$ is a sufficiently smooth positive function with non-degenerate critical points $\xi_{1}, \xi_{2}, \cdots, \xi_{k}$ and satisfies some degree conditions around these points, then there exists a solution of (1.3) concentrating to these $k$ critical points. (See [12] for the case $k=1$ with more technical conditions.) Further, in [20], Fall, Mahmoudi and Valdinoci proved that if there exist semiclassical solutions to (1.3) as $\varepsilon \rightarrow 0$, then the concentration points must be critical points of $V$.

Moreover, we should mention that the concentration phenomena for fractional Schrödinger equations on bounded domain with Dirichlet condition were investigated by Dávila, del Pino, Dipierro and Valdinoci [14].

In this paper, we mainly investigate existence and multiplicity of semiclassical standing wave solutions to Equation (1.3) when $V$ has non-isolated critical points. More precisely, we have the following theorem.

THEOREM 1.1. Let $0<s<1, n>4-4 s$. Suppose that $V$ is a non-negative function in $C_{b}^{3}\left(\mathbf{R}^{n}\right)$ with a non-degenerate smooth compact critical manifold $M$. Then for $\varepsilon>0$ small, Equation (1.3) has at least $l(M)$ solutions concentrating near points of $M$.

Here $l(M)$ denotes the cup length of $M$ (see Section 6.1 below) and

$$
C_{b}^{3}\left(\mathbf{R}^{n}\right)=\left\{v \in C^{3}\left(\mathbf{R}^{n}\right) \mid \partial^{J} v \text { is bounded on } \mathbf{R}^{n} \text { for all }|J| \leq 3\right\} .
$$

The non-degeneracy of a critical manifold is in the sense of Bott [8]. Precisely, we say that a critical manifold $M$ of $V$ is non-degenerate if, for every $x \in M$, the kernel of $D^{2} f(x)$ equals to $T_{x} M$.

REMARK 1.2. When $s=1$, the result of this theorem was obtained by Ambrosetti, Malchiodi and Secchi [4].

REMARK 1.3. Since the unique positive solution (up to translation) to the standard equation decays as $1 /\left(1+|x|^{n+2 s}\right)$ (see for example $[\mathbf{2 4}, \mathbf{2 2}]$ or Theorem 2.3 below), we 
should technically assume that $0<s<1$ and $n>4-4 s$ to make some necessary integrals convergent (see the proof of Lemma 3.2 below). Based on our observation, this assumption is essential since the decay estimate of the unique standard solution is optimal. We should also note that when $s \rightarrow 1$, there is no restriction on the dimension $n$. This is the same as the classical case $s=1$.

REMARK 1.4. Note that the assumption $V \geq 0$ on $\mathbf{R}^{n}$ is not essential. In fact, a similar argument as in Section 2.3 below implies that the condition $\inf (1+V)>0$ is sufficient. Without loss of generality, in what follows we assume that $V(0)=0$ for simplicity.

Our proof relies on a singular perturbation argument as in [4]. More precisely, by the change of variable $x \rightarrow \varepsilon x$, Equation (1.3) becomes

$$
(-\Delta)^{s} u+u+V(\varepsilon x) u=|u|^{p-1} u .
$$

Solutions of (1.4) are the critical points $u \in H^{s}\left(\mathbf{R}^{n}\right)$ of the functional

$$
f_{\varepsilon}(u)=f_{0}(u)+\frac{1}{2} \int_{\mathbf{R}^{n}} V(\varepsilon x) u^{2} d x,
$$

where

$$
f_{0}(u)=\frac{1}{2}\|u\|_{s}^{2}-\frac{1}{p+1} \int_{\mathbf{R}^{n}}|u|^{p+1} d x .
$$

Here $\|\cdot\|_{s}$ denotes the norm in $H^{s}\left(\mathbf{R}^{n}\right)$. We should note that $f_{\varepsilon} \in C^{2}\left(H^{s}\left(\mathbf{R}^{n}\right)\right)$. We will find the solutions of (1.4) near the solutions of

$$
(-\Delta)^{s} u+u+V(\varepsilon \xi) u=|u|^{p-1} u,
$$

for some $\xi \in \mathbf{R}^{n}$ to be fixed. The solutions of (1.7) are critical points of the following functional

$$
F_{\varepsilon, \xi}(u)=f_{0}(u)+\frac{1}{2} V(\varepsilon \xi) \int_{\mathbf{R}^{n}} u^{2} d x .
$$

Since (1.8) has a term of $V, F_{\varepsilon, \xi}$ inherits the topological features of the critical manifold $M$ of $V$. Therefore, if we consider $f_{\varepsilon}$ as a perturbation of $F_{\varepsilon, \xi}$, multiple solutions to (1.4) will be found by a multiplicity theorem from [11] (see Theorem 6.1 below).

Nevertheless, a direct application of the arguments in [4] to our problem is impossible. There are two reasons which make our proof much more complicated. Firstly, unlike the Laplacian $-\Delta$, the fractional Laplacian $(-\Delta)^{s}, 0<s<1$, is nonlocal. For this reason, when $0<s<1$, the classical local techniques as in $s=1$ case (see [4]) can not be used any more. For instance, instead of using the classical method in [4] which depends on the locality of $-\Delta$ essentially, we employ a functional analysis approach to prove the invertibility of $D^{2} f_{\varepsilon}$ (see Section 4 below). Secondly, the standard solution $U$ to unperturbed fractional Schrödinger equation $(V \equiv 0$ in Equation (1.3)) decays only as $1 /\left(1+|x|^{n+2 s}\right)$ (see Section 2.2 below), especially it does not decay exponentially as in $s=1$ case. Therefore, to ensure the necessary functions in certain Sobolev spaces on $\mathbf{R}^{n}$ and to recover the estimates for Lyapunov-Schmidt reduction, we need more detailed and involved analysis than the classical case (see Section 3, 4 and 5 below). 
Our paper is organized as follows. In Section 2, we recall the notations of Fractional Sobolev spaces, some basic properties of standard equation which is obtained by $[\mathbf{2 5}, \mathbf{2 4}$, 22]. Moreover, we formulate the functional corresponding Equation (1.3), and construct the critical manifold of the functional (1.8). In Section 3, some useful estimates are showed for further reference. In Section 4, we prove the invertibility of linearized operator at the points on critical manifold of $F_{\varepsilon, \xi}$. In Section 5, we apply the Lyapunov-Schmidt reduction method to our functional. In Section 6, we complete the proof of Theorem 1.1.

\section{Preliminaries}

In this section, we recall some results on fractional Laplacian, fractional Sobolev spaces and some uniqueness, non-degeneracy and decay results for solutions to the standard Schrödinger equations.

2.1. Fractional Laplacian and fractional order Sobolev spaces. For further references, we recall some basic facts involving fractional Laplacian and fractional order Sobolev spaces. For more details, see, for example, [1], [35], [19], [10].

Mathematically, $(-\Delta)^{s}$ is defined as

$$
(-\Delta)^{s} u=C(n, s) \text { P.V. } \int_{\mathbf{R}^{n}} \frac{u(x)-u(y)}{|x-y|^{n+2 s}} d y=C(n, s) \lim _{\delta \rightarrow 0^{+}} \int_{\mathbf{R}^{n} \backslash B_{\delta}(x)} \frac{u(x)-u(y)}{|x-y|^{n+2 s}} d y .
$$

Here P. V. is a commonly used abbreviation for 'in the principal value sense' and $C(n, s)=$ $\pi^{-(2 s+n / 2)} \frac{\Gamma(n / 2+s)}{\Gamma(-s)}$. It is well known that $(-\Delta)^{s}$ on $\mathbf{R}^{n}$ with $s \in(0,1)$ is a nonlocal operator.

When $s \in(0,1)$, the space $H^{s}\left(\mathbf{R}^{n}\right)=W^{s, 2}\left(\mathbf{R}^{n}\right)$ is defined by

$$
\begin{aligned}
H^{s}\left(\mathbf{R}^{n}\right) & =\left\{u \in L^{2}\left(\mathbf{R}^{2}\right): \frac{|u(x)-u(y)|}{|x-y|^{\frac{n}{2}+s}} \in L^{2}\left(\mathbf{R}^{n} \times \mathbf{R}^{n}\right)\right\} \\
& =\left\{u \in L^{2}\left(\mathbf{R}^{2}\right): \int_{\mathbf{R}^{n}}\left(1+|\zeta|^{2 s}\right)|\mathcal{F} u(\zeta)|^{2} d \zeta<+\infty\right\}
\end{aligned}
$$

and the inner product is

$$
\langle u, v\rangle_{s}:=\int_{\mathbf{R}^{n}} u v d x+\int_{\mathbf{R}^{n}} \int_{\mathbf{R}^{n}} \frac{(u(x)-u(y))(v(x)-v(y))}{|x-y|^{n+2 s}} d x d y .
$$

Let

$$
[u]_{s}:=[u]_{H^{s}\left(\mathbf{R}^{n}\right)}=\left(\int_{\mathbf{R}^{n}} \int_{\mathbf{R}^{n}} \frac{|u(x)-u(y)|^{2}}{|x-y|^{n+2 s}} d x d y\right)^{\frac{1}{2}}
$$

be the Gagliardo (semi) norm of $u$. The following identity yields the relation between the fractional operator $(-\Delta)^{s}$ and the fractional Sobolev space $H^{s}\left(\mathbf{R}^{n}\right)$,

$$
[u]_{H^{s}\left(\mathbf{R}^{n}\right)}=C\left(\int_{\mathbf{R}^{n}}|\zeta|^{2 s}|\mathcal{F} u(\zeta)|^{2} d \zeta\right)^{\frac{1}{2}}=C\left\|(-\Delta)^{\frac{s}{2}} u\right\|_{L^{2}\left(\mathbf{R}^{n}\right)}
$$

for a suitable positive constant $C$ depending only on $s$ and $n$.

When $s>1$ and it is not an integer we write $s=m+\sigma$, where $m$ is an integer and $\sigma \in(0,1)$. In this case the space $H^{s}\left(\mathbf{R}^{n}\right)$ consists of those equivalence classes of functions 
$u \in H^{m}\left(\mathbf{R}^{n}\right)$ whose distributional derivatives $D^{J} u$, with $|J|=m$, belong to $H^{\sigma}\left(\mathbf{R}^{n}\right)$, namely

$$
H^{s}\left(\mathbf{R}^{n}\right)=\left\{u \in H^{m}\left(\mathbf{R}^{n}\right): D^{J} u \in H^{\sigma}\left(\mathbf{R}^{n}\right) \text { for any } J \text { with }|J|=m\right\}
$$

and this is a Banach space with respect to the norm

$$
\|u\|_{s}:=\|u\|_{H^{s}\left(\mathbf{R}^{n}\right)}=\left(\|u\|_{H^{m}\left(\mathbf{R}^{n}\right)}^{2}+\sum_{|J|=m}\left\|D^{J} u\right\|_{H^{\sigma}\left(\mathbf{R}^{n}\right)}^{2}\right)^{\frac{1}{2}} .
$$

Clearly, if $s=m$ is an integer, the space $H^{s}\left(\mathbf{R}^{n}\right)$ coincides with the usual Sobolev space $H^{m}\left(\mathbf{R}^{n}\right)$. By this notation, we denote the norm of $L^{2}\left(\mathbf{R}^{n}\right)$ by $\|\cdot\|_{0}$.

For a general domain $\Omega$, the space $H^{s}(\Omega)$ can be defined similarly.

On the Sobolev inequality and the compactness of embedding, one has

Theorem 2.1. [1] Let $\Omega$ be a domain with smooth boundary in $\mathbf{R}^{n}$. Let $s>0$, then

(a) If $n>2 s$, then $H^{s}(\Omega) \hookrightarrow L^{r}(\Omega)$ for $2 \leq r \leq 2 n /(n-2 s)$,

(b) If $n=2 s$, then $H^{s}(\Omega) \hookrightarrow L^{r}(\Omega)$ for $2 \leq r<\infty$,

THEOREM 2.2. [35] Let $s>s^{\prime}$ and $\Omega$ be a bounded domain with smooth boundary in $\mathbf{R}^{n}$. Then the embedding operator

$$
i_{s}^{s^{\prime}}: H^{s}(\Omega) \rightarrow H^{s^{\prime}}(\Omega)
$$

is compact.

2.2. Some results for the standard equation. We recall some basic properties of the solutions to the following equation

$$
(-\Delta)^{s} u+u-|u|^{p-1} u=0 .
$$

The solutions of (2.1) are the critical points of $f_{0}$ given by (1.6). The non-degeneracy of the standard solution to Equation (2.1) is investigated by many works. For our purpose, we recall the following theorem. (For more results and details on this topic, see, for example, $[\mathbf{2 4}],[\mathbf{2 5}],[\mathbf{2 2}],[\mathbf{2 1}]$ and the references therein.)

THEOREM 2.3. There exists a unique solution (up to translation) $U \in H^{2 s+1}\left(\mathbf{R}^{n}\right)$ to (2.1) such that

$$
\frac{C_{1}}{1+|x|^{n+2 s}} \leq U(x) \leq \frac{C_{2}}{1+|x|^{n+2 s}}, \quad \text { for } x \in \mathbf{R}^{n} \text {, }
$$

with some constants $0<C_{1} \leq C_{2}$. Moreover, the linearized operator $L_{0}$ at $U$ is nondegenerate, that is, its kernel is given by

$$
\operatorname{ker} L_{0}=\operatorname{span}\left\{\partial_{x_{1}} U, \cdots, \partial_{x_{n}} U\right\} .
$$

Remark 2.4. By Lemma C.2 of [24], $\nabla U$ satisfies

$$
|\nabla U(x)| \leq \frac{C}{1+|x|^{n+2 s}},
$$

for some constant $C$. 
REMARK 2.5. The non-degeneracy of $L_{0}$ yields the coercivity estimate as follows:

$$
\left\langle L_{0} \phi, \phi\right\rangle_{0} \geq C\|\phi\|_{s}^{2} \quad \text { for } \phi \perp K,
$$

where $C$ is a positive constant, and $K$ is a suitable chosen $(n+1)$-dimensional subspace. For example, we can choose $K=\operatorname{span}\left\{\phi_{-1}, \partial_{x_{1}} U, \cdots, \partial_{x_{n}} U\right\}$ with $\phi_{-1}$ being the linear ground state of $L_{0}$. For more details, see [24, Section 3].

2.3. Critical points of $F_{\varepsilon, \xi}$. Let

$$
a=a(\xi)=(1+V(\xi))^{\frac{1}{2 s}}
$$

and

$$
b=b(\xi)=[1+V(\xi)]^{\frac{1}{p-1}} .
$$

Then $b U(a x)$ solves (1.7). Set

$$
z^{\varepsilon \xi}=b(\varepsilon \xi) U(a(\varepsilon \xi) x)
$$

and

$$
Z^{\varepsilon}=\left\{z^{\varepsilon \xi}(x-\xi) \mid \xi \in \mathbf{R}^{n}\right\} .
$$

Therefore, every point in $Z^{\varepsilon}$ is a critical point of (1.8) or, equivalently, a solution to Equation (1.7). For simplicity, we will set $z=z_{\xi}=z_{\varepsilon, \xi}=z^{\varepsilon \xi}(x-\xi)$.

\section{Some estimates}

In this section, we prove some useful estimates for future reference. From now on, $C$ denotes various constants.

LEMma 3.1. Let $\bar{\rho}>0$. For $\varepsilon$ sufficiently small and $|\xi| \leq \bar{\rho}$, there holds

$$
\partial_{\xi_{i}} z^{\varepsilon \xi}=-\partial_{x_{i}} z^{\varepsilon \xi}(x-\xi)+O(\varepsilon), \quad \text { in } H^{s}\left(\mathbf{R}^{n}\right) .
$$

Proof. A direct calculation gives

$$
\begin{aligned}
& \partial_{\xi_{i}} z^{\varepsilon \xi}(x-\xi)=\partial_{\xi_{i}}[b(\varepsilon \xi) U(a(\varepsilon \xi)(x-\xi))] \\
= & \varepsilon\left[\partial_{\xi_{i}} b\right](\varepsilon \xi) U(a(\varepsilon \xi)(x-\xi))+\varepsilon b(\varepsilon \xi)\left[\partial_{\xi_{i}} a\right](\varepsilon \xi)[\nabla U](a(\varepsilon \xi)(x-\xi)) \cdot(x-\xi) \\
& -a(\varepsilon \xi) b(\varepsilon \xi)\left[\partial_{x_{i}} U\right](a(\varepsilon \xi)(x-\xi)):=Z_{1}+Z_{2}+Z_{3} .
\end{aligned}
$$

Note that

$$
Z_{3}=-a(\varepsilon \xi) b(\varepsilon \xi)\left[\partial_{x_{i}} U\right](b(\varepsilon \xi)(x-\xi))=-\partial_{x_{i}} z^{\varepsilon \xi}(x-\xi) .
$$

By the definition of $a, b$ and assumption of $V$, we have that

$$
|a(\varepsilon \xi)| \leq C, \quad|b(\varepsilon \xi)|<C, \quad\left|\left[\partial_{\xi_{i}} a\right](\varepsilon \xi)\right| \leq C, \quad\left|\left[\partial_{\xi_{i}} b\right](\varepsilon \xi)\right|<C
$$

for some constant $C$. From assumption of $V$,

$$
|a(\varepsilon \xi)| \geq 1, \quad|b(\varepsilon \xi)| \geq 1 .
$$

Therefore, from $\partial_{x_{i}} U(\cdot-\xi) \in H^{s}\left(\mathbf{R}^{n}\right)$, we have $Z_{3} \in H^{s}\left(\mathbf{R}^{n}\right)$. By $U \in H^{s}\left(\mathbf{R}^{n}\right)$, it holds that

$$
\left\|Z_{1}\right\|_{s}=O(\varepsilon)\left\|\left[\partial_{\xi_{i}} b\right](\varepsilon \xi) U(a(\varepsilon \xi)(\cdot-\xi))\right\|_{s}=O(\varepsilon) .
$$


Since $\partial_{\xi_{i}} \varepsilon^{\varepsilon \xi} \in H^{s}\left(\mathbf{R}^{n}\right)$ and $Z_{1}, Z_{3} \in H^{s}\left(\mathbf{R}^{n}\right)$, we have that $Z_{2}$ is also in $H^{s}\left(\mathbf{R}^{n}\right)$. It follows that $[\nabla U](a(\varepsilon \xi)(\cdot-\xi)) \cdot(\cdot-\xi) \in H^{s}\left(\mathbf{R}^{n}\right)$. So, we obtain that $[\nabla U](\cdot-\xi) \cdot(\cdot-\xi) \in H^{s}\left(\mathbf{R}^{n}\right)$. Again, by the property of $a$, it holds that

$$
\left\|Z_{2}\right\|_{s}=O(\varepsilon) .
$$

From (3.2) and (3.3), we have (3.1). This completes the proof.

Lemma 3.2. Given $\bar{\rho}>0$ and small $\bar{\varepsilon}>0$, we have that, if $|\xi| \leq \bar{\rho}$ and $0<\varepsilon<\bar{\varepsilon}$, then

and

$$
\int_{\mathbf{R}^{n}}|V(\varepsilon x)-V(\varepsilon \xi)|^{2} z_{\xi}^{2} d x \leq C\left(\varepsilon^{2}|\nabla V(\varepsilon \xi)|^{2}+\varepsilon^{4}\right)
$$

$$
\int_{\mathbf{R}^{n}}|V(\varepsilon x)-V(\varepsilon \xi)|^{2}\left|\partial_{x_{i}} z_{\xi}\right|^{2} d x \leq C\left(\varepsilon^{2}|\nabla V(\varepsilon \xi)|^{2}+\varepsilon^{4}\right) .
$$
that

Proof. Since $V \in C_{b}^{3}\left(\mathbf{R}^{n}\right)$ implies that $|\nabla V(x)| \leq C$ and $\left|D^{2} V(x)\right| \leq C$, it holds

$$
|V(\varepsilon x)-V(\varepsilon \xi)| \leq \varepsilon|\nabla V(\varepsilon \xi)| \cdot|x-\xi|+C \varepsilon^{2}|x-\xi|^{2} .
$$

Therefore,

$$
\begin{aligned}
\int_{\mathbf{R}^{n}}|V(\varepsilon x)-V(\varepsilon \xi)|^{2} z_{\xi}^{2} d x \leq & C \varepsilon^{2}|\nabla V(\varepsilon \xi)|^{2} \int_{\mathbf{R}^{n}}|x-\xi|^{2} z_{\xi}^{2}(x-\xi) d x \\
& +C \varepsilon^{4} \int_{\mathbf{R}^{n}}|x-\xi|^{4} z_{\xi}^{2}(x-\xi) d x .
\end{aligned}
$$

By the definition of $z_{\xi}$,

$$
\begin{aligned}
\int_{\mathbf{R}^{n}}|x-\xi|^{2} z_{\xi}^{2}(x-\xi) d x & =b^{2}(\varepsilon \xi) \int_{\mathbf{R}^{n}}|y|^{2} U^{2}(a(\varepsilon \xi) y) d y \\
& =a^{-n-2} b^{2} \int_{\mathbf{R}^{n}}\left|y^{\prime}\right|^{2} U^{2}\left(y^{\prime}\right) d y^{\prime}
\end{aligned}
$$

Using Theorem 2.3, we obtain

$$
\int_{\mathbf{R}^{n}}\left|y^{\prime}\right|^{2} U^{2}\left(y^{\prime}\right) d y^{\prime} \leq C_{2} \int_{\mathbf{R}^{n}} \frac{\left|y^{\prime}\right|^{2}}{\left(1+\left|y^{\prime}\right|\right)^{2 n+4 s}} \leq C .
$$

Since we assume $n>4-4 s$, it follows that

$$
\begin{aligned}
\int_{\mathbf{R}^{n}}|x-\xi|^{4} z_{\xi}^{2}(x-\xi) d x & \leq C_{2} \int_{\mathbf{R}^{n}} \frac{|x-\xi|^{4}}{(1+|x-\xi|)^{2 n+4 s}} d x \\
& \leq C_{2} \int_{\mathbf{R}^{n}} \frac{1}{(1+|x-\xi|)^{2 n+4 s-4}} d x \leq C .
\end{aligned}
$$

Therefore, we get

$$
\int_{\mathbf{R}^{n}}|V(\varepsilon x)-V(\varepsilon \xi)|^{2} z_{\xi}^{2} d x \leq C\left(\varepsilon^{2}|\nabla V(\varepsilon \xi)|^{2}+\varepsilon^{4}\right) .
$$


For the second estimate, we have

$$
\begin{aligned}
\int_{\mathbf{R}^{n}}|V(\varepsilon x)-V(\varepsilon \xi)|^{2}\left|\partial_{x_{i}} z_{\xi}\right|^{2} d x \leq & C \varepsilon^{2}|\nabla V(\varepsilon \xi)|^{2} \int_{\mathbf{R}^{n}}|x-\xi|^{2}\left|\partial_{x_{i}} z_{\xi}(x-\xi)\right|^{2} d x \\
& +C \varepsilon^{4} \int_{\mathbf{R}^{n}}|x-\xi|^{4}\left|\partial_{x_{i}} z_{\xi}(x-\xi)\right|^{2} d x .
\end{aligned}
$$

By Remark 2.4, $\left|\partial_{x_{i}} z_{\xi}(x-\xi)\right| \leq \frac{C}{1+|x-\xi|^{n+2 s}}$. Then a similar argument as the proof of (3.4) gives

$$
\int_{\mathbf{R}^{n}}|V(\varepsilon x)-V(\varepsilon \xi)|^{2}\left|\partial_{x_{i}} z_{\xi}\right|^{2} d x \leq C\left(\varepsilon^{2}|\nabla V(\varepsilon \xi)|^{2}+\varepsilon^{4}\right) .
$$

This completes the proof.

Lemma 3.3. Given $\bar{\rho}>0$ and small $\bar{\varepsilon}>0$, it holds that, for $|\xi| \leq \bar{\rho}$ and $0<\varepsilon<\bar{\varepsilon}$,

$$
\left\|D f_{\varepsilon}\left(z_{\xi}\right)\right\|_{s} \leq C\left(\varepsilon|\nabla V(\varepsilon \xi)|+O\left(\varepsilon^{2}\right)\right)
$$

for some constant $C$.

Proof. Rewrite

$$
f_{\varepsilon}(u)=F^{\varepsilon \xi}(u)+\frac{1}{2} \int_{\mathbf{R}^{n}}(V(\varepsilon x)-V(\varepsilon \xi)) u^{2} d x .
$$

Since $z_{\xi}$ is a critical point of $F^{\varepsilon \xi}$, we get

$$
\begin{aligned}
\left\langle D f_{\varepsilon}\left(z_{\xi}\right), v\right\rangle_{s} & =\left\langle D F^{\varepsilon \xi}\left(z_{\xi}\right), v\right\rangle_{s}+\int_{\mathbf{R}^{n}}(V(\varepsilon x)-V(\varepsilon \xi)) z_{\xi} v d x \\
& =\int_{\mathbf{R}^{n}}(V(\varepsilon x)-V(\varepsilon \xi)) z_{\xi} v d x .
\end{aligned}
$$

By the Hölder inequality, we have

$$
\begin{aligned}
\left|\left\langle D f_{\varepsilon}\left(z_{\xi}\right), v\right\rangle\right|^{2} & \leq\|v\|_{0}^{2} \int_{\mathbf{R}^{n}}|V(\varepsilon x)-V(\varepsilon \xi)|^{2} z_{\xi}^{2} d x \\
& \leq\|v\|_{s}^{2} \int_{\mathbf{R}^{n}}|V(\varepsilon x)-V(\varepsilon \xi)|^{2} z_{\xi}^{2} d x .
\end{aligned}
$$

Then Lemma 3.2 implies (3.5).

\section{Invertibility}

In this section, we will discuss the invertibility of $D^{2} f_{\varepsilon}\left(z_{\xi}\right)$ on $\left(T_{z_{\xi}} Z^{\varepsilon}\right)^{\perp_{s}}$. Here $T_{z_{\xi}} Z^{\varepsilon}$ is the tangent space to $Z^{\varepsilon}$ at $z_{\xi}$, and $\left(T_{z_{\xi}} Z^{\varepsilon}\right)^{\perp_{s}}$ is the orthogonal complement of $T_{z_{\xi}} Z^{\varepsilon}$ in $H^{s}\left(\mathbf{R}^{n}\right)$.

Let

$$
\mathcal{L}_{\varepsilon, \xi}:\left(T_{z_{\xi}} Z^{\varepsilon}\right)^{\perp_{s}} \rightarrow\left(T_{z_{\xi}} Z^{\varepsilon}\right)^{\perp_{s}}
$$

be the tangent operator of $D f_{\varepsilon}$ restricted on $\left(T_{z_{\xi}} Z^{\varepsilon}\right)^{\perp_{s}}$, that is, on $\left(T_{z_{\xi}} Z^{\varepsilon}\right)^{\perp_{s}}$,

$$
\left\langle\mathcal{L}_{\varepsilon, \xi} v, w\right\rangle_{s}=D^{2} f_{\varepsilon}\left(z_{\xi}\right)[v, w] .
$$


The main aim of this section is to prove the following result which implies that $\mathcal{L}_{\varepsilon, \xi}$ is invertible on $\left(T_{z_{\xi}} Z^{\varepsilon}\right)^{\perp_{s}}$.

Proposition 4.1. Given $\bar{\rho}>0$, there exists $\bar{\varepsilon}>0$ such that, for all $|\xi| \leq \bar{\rho}$ and $0<\varepsilon<\bar{\varepsilon}$, it holds that

$$
\left|\left\langle\mathcal{L}_{\varepsilon, \xi} v, v\right\rangle_{s}\right| \geq C\|v\|_{s}^{2}, \quad \forall v \in\left(T_{z_{\xi}} Z^{\varepsilon}\right)^{\perp_{s}}
$$

where $C>0$ is a constant only depending on $\bar{\xi}$ and $\bar{\varepsilon}$.

Note that

$$
T_{z_{\xi}} Z^{\varepsilon}=\operatorname{span}\left\{\partial_{\xi_{1}} z_{\xi}, \cdots, \partial_{\xi_{n}} z_{\xi}\right\}
$$

By Lemma 3.1, we know that $\partial_{\xi_{i}} z_{\xi}$ is close to $-\partial_{x_{i}} z_{\xi}$ in $H^{s}\left(\mathbf{R}^{n}\right)$ when $\varepsilon \rightarrow 0$ and $|\xi| \leq \bar{\rho}$. For convenience, we define

$$
K_{\varepsilon, \xi}=\operatorname{span}\left\{z_{\xi}, \partial_{x_{1}} z_{\xi}, \cdots, \partial_{x_{n}} z_{\xi}\right\} .
$$

To prove Proposition 4.1, we need some lemmas.

LEMMA 4.2. $z_{\xi}$ is a critical point of $F^{\varepsilon \xi}$ with Morse index one.

Proof. Since

$$
D^{2} F^{\varepsilon \xi}\left(z_{\xi}\right)\left[z_{\xi}, z_{\xi}\right]=-(p-1) \int_{\mathbf{R}^{n}} z_{\xi}^{p+1} d x<0,
$$

the operator $D^{2} F^{\varepsilon \xi}\left(z_{\xi}\right)$ has at least one negative eigenvalue. For the details to prove that the Morse index of $z_{\xi}$ is one exactly, see Section 3 in [24].

LEMMA 4.3. Let $\bar{\rho}>0$, there exist $\varepsilon_{0}>0$ and a constant $C_{1}>0$ such that, for all $0<\varepsilon<\varepsilon_{0}$ and all $|\xi| \leq \bar{\rho}$, it holds

$$
\left\langle\mathcal{L}_{\varepsilon, \xi} z_{\xi}, z_{\xi}\right\rangle_{s} \leq-C_{1}<0
$$

Proof. A direct calculus yields

$$
\left\langle\mathcal{L}_{\varepsilon, \xi} z_{\xi}, z_{\xi}\right\rangle_{s}=D^{2} F^{\varepsilon \xi}\left(z_{\xi}\right)\left[z_{\xi}, z_{\xi}\right]+\int_{\mathbf{R}^{n}}(V(\varepsilon x)-V(\varepsilon \xi)) z_{\xi}^{2} d x .
$$

By (4.2) and (2.4),

$$
\begin{aligned}
D^{2} F^{\varepsilon \xi}\left(z_{\xi}\right)\left[z_{\xi}, z_{\xi}\right] & =-(p-1) \int_{\mathbf{R}^{n}} z_{\xi}^{p+1} d x \\
& =-(p-1) \int_{\mathbf{R}^{n}}|b(\varepsilon \xi) U(a(\varepsilon \xi)(x-\xi))|^{p+1} d x \\
& =-(p-1)[b(\varepsilon \xi)]^{p+1}[a(\varepsilon \xi)]^{-n} \int_{\mathbf{R}^{n}} U^{p+1}(x) d x
\end{aligned}
$$

From the definition of $a, b$ (see (2.2) (2.3)) and $V(0)=0$, we have that, for any fixed $\bar{\rho}>0$, there exists $\varepsilon_{1}>0$ small enough such that when $|\xi| \leq \bar{\rho}$ and $0<\varepsilon<\varepsilon_{1}$, it holds

$$
a(\varepsilon \xi) \in\left[\frac{1}{2}, 2\right] \quad \text { and } \quad b(\varepsilon \xi) \in\left[\frac{1}{2}, 2\right] .
$$


Since $U$ is the unique solution (up to translation),

$$
\int_{\mathbf{R}^{n}} U^{p+1}(x) d x
$$

is a constant. Therefore there is a positive constant $C_{0}$ such that

$$
D^{2} F^{\varepsilon \xi}\left(z_{\xi}\right)\left[z_{\xi}, z_{\xi}\right] \leq-C_{0}<0
$$

From Lemma 3.2, the second term on right side of (4.3) satisfies

$$
\begin{aligned}
& \left|\int_{\mathbf{R}^{n}}(V(\varepsilon x)-V(\varepsilon \xi)) z_{\xi}^{2} d x\right| \\
\leq & \int_{\mathbf{R}^{n}}\left(\varepsilon|\nabla V(\varepsilon \xi) \cdot(x-\xi)|+\varepsilon^{2}\left|D^{2} V(\eta)\right| \cdot|x-\xi|^{2}\right) z_{\xi}^{2} d x \\
= & \varepsilon \int_{\mathbf{R}^{n}}|\nabla V(\varepsilon \xi) \cdot(x-\xi)| z_{\xi}^{2} d x+\varepsilon^{2} \int_{\mathbf{R}^{n}}\left|D^{2} V(\eta)\right| \cdot|x-\xi|^{2} z_{\xi}^{2} d x .
\end{aligned}
$$

Here $\eta$ is some point in $\mathbf{R}^{n}$. Since $V \in C_{b}^{3}\left(\mathbf{R}^{n}\right)$, we have that

$$
|\nabla V(\varepsilon \xi) \cdot(x-\xi)| \leq C|x-\xi|,
$$

and

$$
\left|D^{2} V(\eta)\right| \cdot|x-\xi|^{2} \leq C|x-\xi|^{2} .
$$

Then by the definition of $z_{\xi}$,

$$
\begin{aligned}
& \int_{\mathbf{R}^{n}}|\nabla V(\varepsilon \xi) \cdot(x-\xi)| z_{\xi}^{2} d x \\
\leq & C \int_{\mathbf{R}^{n}}|x-\xi||b(\varepsilon \xi) U(a(\varepsilon \xi)(x-\xi))|^{2} d x \\
\leq & C[b(\varepsilon \xi)]^{2}[a(\varepsilon \xi)]^{-n-1} \int_{\mathbf{R}^{n}} \frac{|x-\xi|}{(1+|x-\xi|)^{2 n+4 s}} d x
\end{aligned}
$$

Taking $|\xi| \leq \rho$ and $\varepsilon<\varepsilon_{1}$ as in (4.4), we obtain that there exists a positive constant $C_{2}$ such that

A similar argument yields

$$
\int_{\mathbf{R}^{n}}|\nabla V(\varepsilon \xi) \cdot(x-\xi)| z_{\xi}^{2} d x<C_{2}
$$

$$
\int_{\mathbf{R}^{n}}\left|D^{2} V(\eta)\right| \cdot|x-\xi|^{2} z_{\xi}^{2} d x \leq C_{3} \int_{\mathbf{R}^{n}} \frac{|x-\xi|^{2}}{1+|x-\xi|^{2 n+4 s}} d x \leq C_{4} .
$$

Therefore, when $|\xi|<\rho$ and $\varepsilon<\varepsilon_{1}$,

$$
\left|\int_{\mathbf{R}^{n}}(V(\varepsilon x)-V(\varepsilon \xi)) z_{\xi}^{2} d x\right| \leq C_{2} \varepsilon+C_{3} \varepsilon^{2}
$$

Then there is a $\varepsilon_{0}<\varepsilon_{1}$ such that when $\varepsilon<\varepsilon_{0}$,

$$
C_{2} \varepsilon+C_{3} \varepsilon^{2}<\frac{C_{0}}{2}
$$


From (4.5), (4.6), (4.7), we have

$$
\left\langle\mathcal{L}_{\varepsilon, \xi} z_{\xi}, z_{\xi}\right\rangle_{s} \leq-\frac{C_{0}}{2}<0
$$

This complete the proof.

LEMma 4.4. Let $\bar{\rho}>0$. There exists $\varepsilon_{2}>0$ small such that, for all $0<\varepsilon<\varepsilon_{2}$ and $|\xi| \leq \bar{\rho}$, it holds

$$
D^{2} f_{0}\left(z_{\xi}\right)[\phi, \phi] \geq C_{2}\|\phi\|_{s}^{2}, \quad \text { for } \phi \in K_{\varepsilon, \xi}^{\perp_{s}},
$$

where $C_{2}$ is a positive constant only depending on $\varepsilon_{2}$ and $\bar{\rho}$.

If this lemma does not hold, then there exists a sequence of $\left(\varepsilon_{j}, \xi_{j}\right) \rightarrow(0, \bar{\xi})$ in $\mathbf{R}^{+} \times$ $B_{\bar{\rho}} \subset \mathbf{R}^{+} \times \mathbf{R}^{n}$ and a sequence $\phi_{j} \in K_{\xi_{j}, \varepsilon_{j}}^{\perp_{s}}$ such that

$$
\left\|\phi_{j}\right\|_{s}=1 \text {, }
$$

and

$$
D^{2} f_{0}\left(z_{\xi_{j}}\right)\left[\phi_{j}, \phi_{j}\right] \rightarrow 0, \quad \text { as } j \rightarrow \infty .
$$

Since $\left\{\phi_{j}\right\}$ is bounded in $H^{s}\left(\mathbf{R}^{n}\right)$, we assume (passing to a subsequence) that $\phi_{j}$ converge weakly to a $\phi_{\infty}$ in $H^{s}\left(\mathbf{R}^{n}\right)$.

LEMMA 4.5. It holds that

$$
\phi_{\infty} \in K_{0, \bar{\xi}}^{\perp_{s}}
$$

Proof. Rewrite

$$
\begin{aligned}
\partial_{x_{i}} z_{\xi} & =\partial_{x_{i}} U(x-\bar{\xi})+\partial_{x_{i}}\left[b\left(\varepsilon_{j} \xi_{j}\right) U\left(a\left(\varepsilon_{j} \xi_{j}\right)\left(x-\xi_{j}\right)\right)-U(x-\bar{\xi})\right] \\
& :=\partial_{x_{i}} U(x-\bar{\xi})+\psi_{j} .
\end{aligned}
$$

By the definition of $a(\xi)$ and $b(\xi)$ (see (2.2) and (2.3)), it holds that

$$
\left\|\psi_{j}\right\|_{s} \rightarrow 0, \quad \text { as } j \rightarrow \infty \text {. }
$$

From $\phi_{j} \in K_{\xi_{j}, \varepsilon_{j}}^{\perp_{s}}$, it holds that

$$
0=\left\langle\phi_{j}, \partial_{x_{i}} z_{\xi}\right\rangle_{s}=\left\langle\phi_{j}, \partial_{x_{i}} U(\cdot-\bar{\xi})\right\rangle_{s}+\left\langle\phi_{j}, \psi_{j}\right\rangle_{s} \rightarrow\left\langle\phi_{\infty}, \partial_{x_{i}} U(\cdot-\bar{\xi})\right\rangle_{s} .
$$

That is, $\phi_{\infty} \perp \partial_{x_{i}} U(\cdot-\bar{\xi})$. Similarly, we have that $\phi_{\infty} \perp U(\cdot-\bar{\xi})$. Therefore, we obtain $\phi_{\infty} \in K_{0, \bar{\varepsilon}}^{\perp s}$. This completes the proof.

Let

be the operator given by

$$
\mathcal{L}_{j}: H^{s}\left(\mathbf{R}^{n}\right) \rightarrow H^{s}\left(\mathbf{R}^{n}\right)
$$

$$
\left\langle\mathcal{L}_{j} \phi, \psi\right\rangle_{s}=D^{2} f_{0}\left(z_{\xi_{j}}\right)[\phi, \psi], \quad \text { for } \phi, \psi \in H^{s}\left(\mathbf{R}^{n}\right)
$$

and let

$$
\mathcal{L}_{\infty}: H^{s}\left(\mathbf{R}^{n}\right) \rightarrow H^{s}\left(\mathbf{R}^{n}\right)
$$

be the operator defined by

$$
\left\langle\mathcal{L}_{\infty} \phi, \psi\right\rangle_{s}=D^{2} f_{0}(U(\cdot-\bar{\xi}))[\phi, \psi], \quad \text { for } \phi, \psi \in H^{s}\left(\mathbf{R}^{n}\right) .
$$


We now have the following lemma.

LEMMA 4.6. We have that $\phi_{\infty}=0$.

Proof. By (4.8) and (4.9), we get that

$$
\left\langle\mathcal{L}_{j} \phi_{j}, \phi_{j}\right\rangle_{s}=\left\|\phi_{j}\right\|_{s}^{2}-p \int_{\mathbf{R}^{n}} z_{\xi_{j}}^{p-1} \phi_{j}^{2} d x \rightarrow 0
$$

and then

$$
p \int_{\mathbf{R}^{n}} z_{\xi_{j}}^{p-1} \phi_{j}^{2} d x \rightarrow 1 .
$$

Hence, from the definition of $z_{\xi}$ (see Section 2.3), we obtain that

$$
p \int_{\mathbf{R}^{n}} U^{p-1}(x-\bar{\xi}) \phi_{j}^{2} d x \rightarrow 1 .
$$

Moreover, estimate

$$
\begin{aligned}
& \left|\int_{\mathbf{R}^{n}} U^{p-1}(x-\bar{\xi})\left(\phi_{j}^{2}-\phi_{\infty}^{2}\right) d x\right| \\
\leq & \left(\int_{\mathbf{R}^{n}} U^{2(p-1)}(x-\bar{\xi})\left|\phi_{j}(x)-\phi_{\infty}(x)\right|^{2} d x\right)^{\frac{1}{2}}\left\|\phi_{j}+\phi_{\infty}\right\|_{0} \\
\leq & C\left(\int_{\mathbf{R}^{n}} U^{2(p-1)}(x-\bar{\xi})\left|\phi_{j}(x)-\phi_{\infty}(x)\right|^{2} d x\right)^{\frac{1}{2}} .
\end{aligned}
$$

Let $B_{r}(\bar{\xi})$ be the ball centered at $\bar{\xi}$ with radius $r$. Then

$$
\begin{aligned}
& \int_{\mathbf{R}^{n}} U^{2(p-1)}(x-\bar{\xi})\left|\phi_{j}(x)-\phi_{\infty}(x)\right|^{2} d x \\
= & \left(\int_{B_{r}(\bar{\xi})}+\int_{\mathbf{R}^{n} \backslash B_{r}(\bar{\xi})}\right) U^{2(p-1)}(x-\bar{\xi})\left|\phi_{j}(x)-\phi_{\infty}(x)\right|^{2} d x .
\end{aligned}
$$

For all sufficiently small $\epsilon>0$, there exists an $r(\epsilon)$ such that if $r>r(\epsilon)$, then, $U^{2(p-1)}(x-$ $\bar{\xi})<\epsilon$, for all $x \in \mathbf{R}^{n} \backslash B_{r}(\bar{\xi})$. Thus,

$$
\left|\int_{\mathbf{R}^{n} \backslash B_{r}(\bar{\xi})} U^{2(p-1)}(x-\bar{\xi})\right| \phi_{j}(x)-\left.\phi_{\infty}(x)\right|^{2} d x \mid \leq \epsilon\left\|\phi_{j}(x)-\phi_{\infty}(x)\right\|_{0}^{2} .
$$

We now estimate the other term in (4.11). Let $\chi$ be a smooth function satisfying

$$
\chi(x)= \begin{cases}1, & \text { for } x \in B_{r}(\bar{\xi}) \\ 0, & \text { for } x \in \mathbf{R}^{n} \backslash B_{r+1}(\bar{\xi}) .\end{cases}
$$

Then $\left\{\chi \phi_{j}\right\}$ is a bounded sequence in $H^{s}\left(B_{r+1}(\bar{\xi})\right)$. Therefore, there exists a function $\eta \in H^{s}\left(B_{r+1}(\bar{\xi})\right)$ such that, up to a subsequence, $\chi \phi_{j} \rightarrow \eta$. Since the embedding $H^{s}\left(B_{r+1}(\bar{\xi})\right) \hookrightarrow L^{2}\left(B_{r+1}(\bar{\xi})\right)$ is compact, we have $\chi \phi_{j} \rightarrow \eta$ in $L^{2}\left(B_{r+1}(\bar{\xi})\right)$. Then

$$
\left.\phi_{j}\right|_{B_{r}(\bar{\xi})}=\left.\left.\chi \phi_{j}\right|_{B_{r}(\bar{\xi})} \rightarrow \eta\right|_{B_{r}(\bar{\xi})}, \quad \text { in } L^{2}\left(B_{r}(\bar{\xi})\right) .
$$


Since $\phi_{j} \rightarrow \phi_{\infty}$ in $L^{2}\left(B_{r}(\bar{\xi})\right)$, we obtain that

$$
\phi_{j} \rightarrow \phi_{\infty} \quad \text { in } L^{2}\left(B_{r}(\bar{\xi})\right) .
$$

It follows that

$$
\left|\int_{B_{r}(\bar{\xi})} U^{2(p-1)}(x-\bar{\xi})\right| \phi_{j}(x)-\left.\phi_{\infty}(x)\right|^{2} d x \mid \rightarrow 0, \quad \text { as } j \rightarrow \infty .
$$

By the arbitrary of $\epsilon$, we have that

$$
\int_{\mathbf{R}^{n}} U^{2(p-1)}(x-\bar{\xi})\left|\phi_{j}(x)-\phi_{\infty}(x)\right|^{2} d x \rightarrow 0 .
$$

This yields that

$$
\int_{\mathbf{R}^{n}} U^{p-1}(x-\bar{\xi})\left(\phi_{j}^{2}-\phi_{\infty}^{2}\right) d x \rightarrow 0 .
$$

From (4.10) and (4.13), we get that

$$
p \int_{\mathbf{R}^{n}} U^{p-1}(x-\bar{\xi}) \phi_{\infty}^{2} d x=1 .
$$

On the other hand, by $\phi_{j} \rightarrow \phi_{\infty}$ in $H^{s}\left(\mathbf{R}^{n}\right)$, we have that

$$
\left\langle\phi_{\infty}, \phi_{\infty}\right\rangle_{s} \leftarrow\left\langle\phi_{j}, \phi_{\infty}\right\rangle_{s} \leq\left\|\phi_{j}\right\|_{s}\left\|\phi_{\infty}\right\|_{s}=\left\|\phi_{\infty}\right\|_{s} .
$$

It follows that

$$
\left\|\phi_{\infty}\right\|_{s} \leq 1
$$

Therefore, we obtain that

$$
\left\langle\mathcal{L}_{\infty} \phi_{\infty}, \phi_{\infty}\right\rangle_{s}=\left\|\phi_{\infty}\right\|_{s}^{2}-p \int_{\mathbf{R}^{n}} U^{p-1}(x-\bar{\xi}) \phi_{\infty}^{2} d x \leq 0 .
$$

By Theorem 2.3, Remark 2.5 and Lemma 4.5, it holds that

$$
\left\langle\mathcal{L}_{\infty} \phi_{\infty}, \phi_{\infty}\right\rangle_{s} \geq C\left\|\phi_{\infty}\right\|_{s}^{2},
$$

where $C$ is a positive constant. Then we have that

$$
\left\|\phi_{\infty}\right\|_{s}=0 \text {. }
$$

This completes the proof.

Proof of Lemma 4.4. Note that $z_{\xi_{j}}^{p-1}$ decays uniformly to 0 at infinity as $0<\varepsilon_{j}<\bar{\varepsilon}$ and $|\xi| \leq \bar{\rho}$. Then, for any $\epsilon>0$, there exists a sufficiently large $r_{0}>0$ such that, for all $r>r_{0},\left|z_{\xi_{j}}^{p-1}(x)\right|<\epsilon$ when $x \in \mathbf{R}^{n} \backslash B_{r}$. Therefore, from (4.12) and $\phi_{\infty}=0$, we have that

$$
\left.\left.\left|\int_{\mathbf{R}^{n}} z_{\xi_{j}}^{p-1}\right| \phi_{j}\right|^{2} d x\left|\leq C \int_{B_{r}}\right| \phi_{j}\right|^{2} d x+\epsilon \int_{\mathbf{R}^{n} \backslash B_{r}}\left|\phi_{j}\right|^{2} d x \rightarrow \epsilon, \quad \text { as } j \rightarrow \infty .
$$

By the arbitrary of $\epsilon$, we have that

$$
\left.\left|\int_{\mathbf{R}^{n}} z_{\xi_{j}}^{p-1}\right| \phi_{j}\right|^{2} d x \mid \rightarrow 0, \quad \text { as } j \rightarrow \infty .
$$


Moreover, from (4.8) and (4.9), it holds that

$$
0 \leftarrow D^{2} f_{0}\left(z_{\xi_{j}}\right)\left[\phi_{j}, \phi_{j}\right]=\left\|\phi_{j}\right\|_{s}^{2}-p \int_{\mathbf{R}^{n}} z_{\xi_{j}}^{p-1}\left|\phi_{j}\right|^{2} d x \rightarrow 1 .
$$

It is a contradiction. Thus we have Lemma 4.4.

LEMma 4.7. Let $\bar{\rho}>0$. There exists $\varepsilon_{3}>0$ small such that for all $0<\varepsilon<\varepsilon_{3}$ and $|\xi| \leq \bar{\rho}$, it holds

$$
D^{2} f_{\varepsilon}\left(z_{\xi}\right)[\phi, \phi] \geq C_{3}\|\phi\|_{s}^{2}, \quad \text { for } \phi \in K_{\varepsilon, \xi}^{\perp_{s}}
$$

where $C_{3}$ is a positive constant only depending on $\varepsilon_{2}$ and $\bar{\rho}$.

ProOF. By the nonnegativity of $V$ and Lemma 4.4, we have that, for all $\phi \in K_{\varepsilon, \xi}^{\perp}$

$$
\begin{aligned}
D^{2} f_{\varepsilon}\left(z_{\xi}\right)[\phi, \phi] & =D^{2} f_{0}\left(z_{\xi}\right)[\phi, \phi]+\int_{\mathbf{R}^{n}} V(\varepsilon x) \phi^{s} d x \\
& \geq D^{2} f_{0}\left(z_{\xi}\right)[\phi, \phi] \geq C_{0}\|\phi\|_{s}^{2} .
\end{aligned}
$$

Here $0<\varepsilon<\varepsilon_{2}$ and $|\xi| \leq \bar{\rho}$. Letting $\varepsilon_{3}=\varepsilon_{2}$ and $C_{3}=C_{2}$, we obtain the result.

Proof of Proposition 4.1. Let $\bar{\varepsilon}=\varepsilon_{2}$. From Lemma 4.3, Lemma 4.7, Lemma 3.1 and (4.1), we have that, for all $|\xi| \leq \bar{\rho}$ and $0<\varepsilon<\bar{\varepsilon}$,

$$
\left|\left\langle\mathcal{L}_{\varepsilon, \xi} v, v\right\rangle_{s}\right| \geq C\|v\|_{s}^{2}, \quad \forall v \in\left(T_{z_{\xi}} Z^{\varepsilon}\right)^{\perp_{s}},
$$

where $C>0$ is a constant only depending on $\bar{\xi}$ and $\bar{\varepsilon}$. This completes the proof.

\section{Lyapunov-Schmidt Reduction}

In this section, we will prove that the existence of critical points of $f_{\varepsilon}$ can be reduced to find critical points of an auxiliary finite dimensional functional.

5.1. Auxiliary finite dimensional functional. Let $P_{\varepsilon, \xi}$ be the orthogonal projection onto $\left(T_{z_{\xi}} Z^{\varepsilon}\right)^{\perp_{s}}$. Our aim is to find a point $w \in\left(T_{z_{\xi}} Z^{\varepsilon}\right)^{\perp_{s}}$ satisfying

$$
P_{\varepsilon, \xi} D f_{\varepsilon}\left(z_{\xi}+w\right)=0 \text {. }
$$

By expansion, we have that

$$
D f_{\varepsilon}\left(z_{\xi}+w\right)=D f_{\varepsilon}\left(z_{\xi}\right)+D^{2} f_{\varepsilon}\left(z_{\xi}\right)[w]+\mathcal{R}\left(z_{\xi}, w\right)
$$

Here the map $\mathcal{R}\left(z_{\xi}, w\right)$ is given by

$$
\begin{aligned}
\mathcal{R}\left(z_{\xi}, w\right): H^{s} & \rightarrow \mathbf{R} \\
v & \rightarrow \int_{\mathbf{R}^{n}} R\left(z_{\xi}, w\right) v d x
\end{aligned}
$$

where

$$
R\left(z_{\xi}, w\right)=-\left(\left|z_{\xi}+w\right|^{p-1}\left(z_{\xi}+w\right)-\left|z_{\xi}\right|^{p-1} z_{\xi}-p\left|z_{\xi}\right|^{p-1} w\right)
$$

LEMMA 5.1. For all $w_{1}, w_{2} \in B_{1} \subset H^{s}\left(\mathbf{R}^{n}\right)$, it holds that

$$
\left\|\mathcal{R}\left(z_{\xi}, w_{2}\right)-\mathcal{R}\left(z_{\xi}, w_{1}\right)\right\|_{s} \leq C \max \left\{\left\|w_{1}\right\|_{s}^{\sigma},\left\|w_{2}\right\|_{s}^{\sigma}\right\}\left\|w_{2}-w_{1}\right\|_{s} .
$$

where $\sigma=\min \{1, p-1\}, C$ is a constant independent on $w_{1}, w_{2}$. Here $B_{1}$ is the unit ball in $H^{s}\left(\mathbf{R}^{n}\right)$. 
Proof. For all $v \in H^{s}\left(\mathbf{R}^{n}\right)$,

$$
\begin{aligned}
& \left|\left[\mathcal{R}\left(z_{\xi}, w_{2}\right)-\mathcal{R}\left(z_{\xi}, w_{1}\right)\right](v)\right| \\
\leq & \int_{\mathbf{R}^{n}}|| z_{\xi}+\left.w_{2}\right|^{p-1}\left(z_{\xi}+w_{2}\right)-\left|z_{\xi}+w_{1}\right|^{p-1}\left(z_{\xi}+w_{1}\right)-p\left|z_{\xi}\right|^{p-1}\left(w_{2}-w_{1}\right)|| v \mid d x \\
\leq & \left.p \int_{\mathbf{R}^{n}}|| z_{\xi}+w_{1}+\left.\theta_{1}\left(w_{2}-w_{1}\right)\right|^{p-1}-\left|z_{\xi}\right|^{p-1}\right)|| w_{2}-w_{1}|| v \mid d x .
\end{aligned}
$$

Here $\theta_{1} \in[0,1]$. For $1<p \leq 2$,

$$
\begin{aligned}
\left|\left[\mathcal{R}\left(z_{\xi}, w_{2}\right)-\mathcal{R}\left(z_{\xi}, w_{1}\right)\right](v)\right| & \leq p \int_{\mathbf{R}^{n}}\left|w_{1}+\theta_{1}\left(w_{2}-w_{1}\right)\right|^{p-1}\left|w_{2}-w_{1}\right||v| d x \\
& \leq C \int_{\mathbf{R}^{n}}\left(\left|w_{1}\right|+\left|w_{2}\right|\right)^{p-1}\left|w_{2}-w_{1}\right||v| d x \\
& \leq C\left(\left\|w_{1}\right\|_{L^{p+1}}^{p-1}+\left\|w_{2}\right\|_{L^{p+1}}^{p-1}\right)\left\|w_{2}-w_{1}\right\|_{L^{p+1}}\|v\|_{L^{p+1}} .
\end{aligned}
$$

By Sobolev imbedding (Theorem 2.1), we have that

$$
H^{s}\left(\mathbf{R}^{n}\right) \hookrightarrow L^{p+1}\left(\mathbf{R}^{n}\right) .
$$

Therefore, we obtain that

$$
\left|\left[\mathcal{R}\left(z_{\xi}, w_{2}\right)-\mathcal{R}\left(z_{\xi}, w_{1}\right)\right](v)\right| \leq C\left(\left\|w_{1}\right\|_{s}^{p-1}+\left\|w_{2}\right\|_{s}^{p-1}\right)\left\|w_{2}-w_{1}\right\|_{s}\|v\|_{s} .
$$

For $2<p<\frac{n+2 s}{n-2 s}$ (if $2<\frac{n+2 s}{n-2 s}$ ), it holds that

$$
\begin{aligned}
& \left|\left[\mathcal{R}\left(z_{\xi}, w_{2}\right)-\mathcal{R}\left(z_{\xi}, w_{1}\right)\right](v)\right| \\
= & C \int_{\mathbf{R}^{n}}\left|z_{\xi}+\theta_{2}\left(w_{1}+\theta_{1}\left(w_{2}-w_{1}\right)\right)\right|^{p-2}\left|w_{2}-w_{1}\right|^{2}|v| d x \\
\leq & C\left\|z_{\xi}+\theta_{2}\left(w_{1}+\theta_{1}\left(w_{2}-w_{1}\right)\right)\right\|_{L^{p+1}}^{p-2}\left\|w_{2}-w_{1}\right\|_{L^{p+1}}^{2}\|v\|_{L^{p+1}},
\end{aligned}
$$

where $\theta_{2} \in[0,1]$. Similarly, by Sobolev imbedding, we have that

$$
\mid\left[\mathcal{R}\left(z_{\xi}, w_{2}\right)-\mathcal{R}\left(z_{\xi}, w_{1}\right](v) \mid \leq C\left(\left\|z_{\xi}\right\|_{s}+\left\|w_{1}\right\|_{s}+\left\|w_{2}\right\|_{s}\right)^{p-2}\left\|w_{2}-w_{1}\right\|_{s}^{2}\|v\|_{s} .\right.
$$

Therefore, we have

$$
\left\|\mathcal{R}\left(z_{\xi}, w_{2}\right)-\mathcal{R}\left(z_{\xi}, w_{1}\right)\right\|_{s} \leq C \max \left(\left\|w_{1}\right\|_{s}^{\sigma},\left\|w_{1}\right\|_{s}^{\sigma}\right)\left\|w_{2}-w_{1}\right\|_{s},
$$

where $\sigma=\min \{1, p-1\}$. This completes the proof.

Corollary 5.2. It holds that $\left\|\mathcal{R}\left(z_{\xi}, w\right)\right\|_{s}=O\left(\|w\|_{s}^{1+\sigma}\right)$ where $\sigma=\min \{1, p-1\}$.

Proof. Choosing $w_{1}=0$ and $w_{2}=w$ in Lemma 5.1, we find that

$$
\left\|\mathcal{R}\left(z_{\xi}, w\right)\right\|_{s} \leq C\left(\|w\|_{s}^{1+\sigma}\right)
$$


From the definition of $\mathcal{L}_{\varepsilon, \xi}$, Equation (5.1) becomes

$$
\mathcal{L}_{\varepsilon, \xi} w+P_{\varepsilon, \xi} D f_{\varepsilon}\left(z_{\xi}\right)+P_{\varepsilon, \xi} \mathcal{R}\left(z_{\xi}, w\right)=0, \quad \text { for } w \in\left(T_{z_{\xi}} Z\right)^{\perp_{s}} .
$$

By Proposition 4.1, we known that $\mathcal{L}_{\varepsilon, \xi}$ is invertible on $\left(T_{z_{\xi}} Z\right)^{\perp_{s}}$. Denote the invertible operator by $\mathcal{L}_{\varepsilon, \xi}^{-1}$. Then Equation (5.2) is equivalent to

$$
w=N_{\varepsilon, \xi}(w) .
$$

Here

$$
N_{\varepsilon, \xi}(w)=-\mathcal{L}_{\varepsilon, \xi}^{-1}\left(P_{\varepsilon \xi} D f_{\varepsilon}\left(z_{\xi}\right)+P_{\varepsilon \xi} R\left(z_{\xi}, w\right)\right) .
$$

Lemma 5.3. There is a small ball $B_{\delta} \subset\left(T_{z_{\xi}} Z^{\varepsilon}\right)^{\perp_{s}}$ such that $N_{\varepsilon, \xi}$ maps $B_{\delta}$ to itself if $0<\varepsilon<\bar{\varepsilon}$ and $|\xi| \leq \bar{\rho}$.

Proof. Using Lemma 3.3, we obtain

$$
\left\|N_{\varepsilon, \xi}(w)\right\|_{s} \leq C\left(\varepsilon|\nabla V(\varepsilon \xi)|+O\left(\varepsilon^{2}\right)\right)+O\left(\|w\|_{s}^{1+\sigma}\right) .
$$

Then there is a small positive constant $\delta$ such that $N_{\varepsilon, \xi}$ maps $B_{\delta} \subset\left(T_{z_{\xi}} Z\right)^{\perp_{s}}$ to itself if $0<\varepsilon<\bar{\varepsilon}$ and $|\xi| \leq \bar{\rho}$.

Lemma 5.4. For all $w_{1}, w_{2} \in B_{1} \subset H^{s}\left(\mathbf{R}^{n}\right)$, we have that

$$
\left\|N_{\varepsilon, \xi}\left(w_{2}\right)-N_{\varepsilon, \xi}\left(w_{1}\right)\right\|_{s} \leq C \max \left(\left\|w_{1}\right\|_{s}^{\sigma},\left\|w_{2}\right\|_{s}^{\sigma}\right)\left\|w_{2}-w_{1}\right\|_{s},
$$

where $C$ is a constant independent on $w_{1}$ and $w_{2}, \sigma=\min \{1, p-1\}$.

Proof. Compute

$$
\begin{aligned}
\left\|N_{\varepsilon, \xi}\left(w_{2}\right)-N_{\varepsilon, \xi}\left(w_{1}\right)\right\|_{s} & =\left\|-\mathcal{L}_{\varepsilon, \xi}^{-1} P_{\varepsilon \xi}\left(\mathcal{R}\left(z_{\xi}, w_{2}\right)-\mathcal{R}\left(z_{\xi}, w_{1}\right)\right)\right\|_{s} \\
& \leq C\left\|\mathcal{R}\left(z_{\xi}, w_{2}\right)-\mathcal{R}\left(z_{\xi}, w_{1}\right)\right\|_{s} .
\end{aligned}
$$

Then by Lemma 5.1, we have that

$$
\left\|N_{\varepsilon, \xi}\left(w_{2}\right)-N_{\varepsilon, \xi}\left(w_{1}\right)\right\|_{s} \leq C \max \left(\left\|w_{1}\right\|_{s}^{\sigma},\left\|w_{1}\right\|_{s}^{\sigma}\right)\left\|w_{2}-w_{1}\right\|_{s},
$$

where $\sigma=\min \{1, p-1\}$. This completes the proof.

Proposition 5.5. For $0<\varepsilon<\bar{\varepsilon}$ and $|\xi| \leq \bar{\rho}$, there exists a unique $w=w(\varepsilon, \xi) \in$ $\left(T_{z_{\xi}} Z\right)^{\perp_{s}}$ such that $D f_{\varepsilon}\left(z_{\xi}+w\right) \in T_{z_{\xi}} Z$, and $w(\varepsilon, \xi)$ is of class $C^{1}$. Moreover, the functional $\Phi_{\varepsilon}(\xi)=f_{\varepsilon}\left(z_{\xi}+w(\varepsilon, \xi)\right)$ has the same regularity as $w$ and satisfies:

$$
\nabla \Phi_{\varepsilon}\left(\xi_{0}\right)=0 \quad \Rightarrow \quad D f_{\varepsilon}\left(z_{\xi_{0}}+w\left(\varepsilon, \xi_{0}\right)\right)=0
$$

Proof. From Lemma 5.3 and 5.4, the map $N_{\varepsilon, \xi}$ is a contraction on $B_{\delta}$ for $0<\varepsilon<\bar{\varepsilon}$ and $|\xi| \leq \bar{\rho}$. Then there exists a unique $w$ such that $w=N_{\varepsilon, \xi}(w)$. For fixed $\varepsilon$, define

$$
\Xi_{\varepsilon}:(\xi, w) \rightarrow P_{\varepsilon, \xi} D f_{\varepsilon}\left(z_{\xi}+w\right) .
$$

Applying the Implicit Function Theorem to $\Xi_{\varepsilon}$, we have that $w(\varepsilon, \xi)$ is $C^{1}$ with respect to $\xi$. Then using a standard argument in $[3, \mathbf{2}]$, we obtain that the critical points of $\Phi_{\varepsilon}(\xi)=f_{\varepsilon}\left(z_{\xi}+w(\varepsilon, \xi)\right)$ give rise to critical points of $f_{\varepsilon}$. 
In what follows, we use the simple notation $w$ to denote $w(\varepsilon, \xi)$ which is obtained in Proposition 5.5.

REMARK 5.6. By Equation (5.3), it follows that

$$
\|w\|_{s} \leq C\left(\varepsilon|\nabla V(\varepsilon \xi)|+\varepsilon^{2}\right)
$$

where $C>0$.

Lemma 5.7. The following inequality holds:

$$
\left\|\nabla_{\xi} w\right\|_{s} \leq C\left(\varepsilon|\nabla V(\varepsilon \xi)|+O\left(\varepsilon^{2}\right)\right)^{\sigma},
$$

where $C>0$ and $\sigma=\min \{1, p-1\}$.

Proof. By (5.2) and Proposition 5.5, we have that, for all $v \in\left(T_{z_{\xi}} Z^{\varepsilon}\right)^{\perp_{s}}$,

$$
\left\langle\mathcal{L}_{\varepsilon, \xi} w, v\right\rangle_{s}+\left\langle D f_{\varepsilon}\left(z_{\xi}\right), v\right\rangle_{s}+\left\langle\mathcal{R}\left(z_{\xi}, w\right), v\right\rangle_{s}=0
$$

Since $D F_{\varepsilon, \xi}\left(z_{\xi}\right)=0$, Equation (5.4) becomes

$$
\begin{aligned}
\langle w, v\rangle_{s}+\int_{\mathbf{R}^{n}} V(\varepsilon x) w v d x-p \int_{\mathbf{R}^{n}} z_{\xi}^{p-1} w v d x+\int_{\mathbf{R}^{n}}[V(\varepsilon x)-V(\varepsilon \xi)] z_{\xi} v d x & \\
& +\int_{\mathbf{R}^{n}} R\left(z_{\xi}, w\right) v d x=0 .
\end{aligned}
$$

Hence

$$
\begin{aligned}
& \left\langle\partial_{\xi_{j}} w, v\right\rangle_{s}+\int_{\mathbf{R}^{n}} V(\varepsilon x)\left(\partial_{\xi_{j}} w\right) v d x-p \int_{\mathbf{R}^{n}} z_{\xi}^{p-1}\left(\partial_{\xi_{j}} w\right) v d x \\
& -p(p-1) \int_{\mathbf{R}^{n}} z_{\xi}^{p-2}\left(\partial_{\xi_{j}} z\right) w v d x+\int_{\mathbf{R}^{n}}(V(\varepsilon x)-V(\varepsilon \xi))\left(\partial_{\xi_{j}} z\right) v d x \\
& -\varepsilon\left(\partial_{x_{j}} V\right)(\varepsilon \xi) \int_{\mathbf{R}^{n}} z v d x-\int_{\mathbf{R}^{n}}\left(R_{z} \partial_{\xi_{j}} z+R_{w} \partial_{\xi_{j}} w\right) v d x=0 .
\end{aligned}
$$

Set $\hat{\mathcal{L}}=\mathcal{L}_{\varepsilon, \xi}-\mathcal{R}_{w}$, where $\left\langle\mathcal{R}_{w} v_{1}, v_{2}\right\rangle=\int_{\mathbf{R}^{n}} R_{w} v_{1} v_{2} d x$. Since $R_{w} \rightarrow 0$ as $w \rightarrow 0$ and $\mathcal{L}_{\varepsilon, \xi}$ is invertible on $\left(T_{z_{\xi}} Z^{\varepsilon}\right)^{\perp_{s}}, \hat{\mathcal{L}}$ is also invertible for $0<\varepsilon<\bar{\varepsilon}$ and $|\xi| \leq \bar{\rho}$. From (5.5), it holds that

$$
\begin{aligned}
\left\langle\hat{\mathcal{L}} \partial_{\xi_{j}} w, v\right\rangle=p(p-1) & \int_{\mathbf{R}^{n}} z_{\xi}^{p-2}\left(\partial_{\xi_{j}} z\right) w v d x-\int_{\mathbf{R}^{n}}(V(\varepsilon x)-V(\varepsilon \xi))\left(\partial_{\xi_{j}} z\right) v d x \\
& +\varepsilon\left(\partial_{x_{j}} V\right)(\varepsilon \xi) \int_{\mathbf{R}^{n}} z v d x+\int_{\mathbf{R}^{n}} R_{z} \partial_{\xi_{j}} z v d x=T_{1}+T_{2}+T_{3}+T_{4} .
\end{aligned}
$$


Next, we shall estimate every term on the left of the equation above. By Theorem 2.3 and Remark 2.4, it holds that, for $1<p \leq 2$,

$$
\begin{aligned}
\left|T_{1}\right| & =p(p-1)\left|\int_{\mathbf{R}^{n}} z_{\xi}^{p-2}\left(\partial_{\xi_{j}} z\right) w v d x\right| \\
& \leq C \int_{\mathbf{R}^{n}}\left(1+|x|^{n+2 s}\right)^{2-p} \frac{1}{1+|x|^{n+2 s}}|w v| d x \\
& \leq C \int_{\mathbf{R}^{n}} \frac{1}{\left(1+|x|^{n+2 s}\right)^{p-1}}|w v| d x \\
& \leq C \int_{\mathbf{R}^{n}}|w v| d x \leq C\|w\|_{0}\|v\|_{0} \leq C\|w\|_{s}\|v\|_{s},
\end{aligned}
$$

and, for $2<p<\frac{n+2 s}{n-2 s}$ (if $2<\frac{n+2 s}{n-2 s}$ ),

$$
\begin{aligned}
\left|\int_{\mathbf{R}^{n}} z_{\xi}^{p-2}\left(\partial_{\xi_{j}} z\right) w v d x\right| & \leq C \int_{\mathbf{R}^{n}} \frac{1}{\left(1+|x|^{n+2 s}\right)^{p-1}}|w v| d x \\
& \leq C\|w\|_{s}\|v\|_{s} .
\end{aligned}
$$

Therefore, we have that

$$
\left|T_{1}\right| \leq C\|w\|_{s}\|v\|_{s}
$$

Since $0<\varepsilon<\bar{\varepsilon}$ and $|\xi| \leq \bar{\rho}$, by Lemma 3.2 we have

$$
\begin{aligned}
\left|T_{2}\right| & =\left|\int_{\mathbf{R}^{n}}(V(\varepsilon x)-V(\varepsilon \xi))\left(\partial_{\xi_{j}} z\right) v d x\right| \\
& \leq \int_{\mathbf{R}^{n}}|V(\varepsilon x)-V(\varepsilon \xi)|\left|\partial_{\xi_{j}} z\right||v| d x \\
& \leq\left(\int_{\mathbf{R}^{n}}|V(\varepsilon x)-V(\varepsilon \xi)|^{2}\left|\partial_{\xi_{j}} z\right|^{2} d x\right)^{\frac{1}{2}}\|v\|_{0} \\
& \leq C \varepsilon|\nabla V(\varepsilon \xi)|\|v\|_{s} .
\end{aligned}
$$

Then we obtain that

$$
\left|T_{2}\right| \leq C \varepsilon|\nabla V(\varepsilon \xi)|\|v\|_{s}
$$

Estimating the third term, we have

$$
\left|T_{3}\right|=\varepsilon\left|\left(\partial_{x_{j}} V\right)(\varepsilon \xi) \int_{\mathbf{R}^{n}} z v d x\right| \leq \varepsilon|(\nabla V)(\varepsilon \xi)|\|z\|_{0}\|v\|_{0} \leq \varepsilon|(\nabla V)(\varepsilon \xi)|\|v\|_{s} .
$$

It remains to estimate the final term. A direct computation yields

$$
\begin{aligned}
\left|T_{4}\right|= & \left|\int_{\mathbf{R}^{n}} R_{z} \partial_{\xi_{j}} z v d x\right| \leq \int_{\mathbf{R}^{n}}\left|R_{z}\right|\left|\partial_{\xi_{j}} z\right||v| d x \\
\leq & C \int_{\mathbf{R}^{n}}|| z_{\xi}+\left.w\right|^{p-1}-\left|z_{\xi}\right|^{p-1}|\cdot| \partial_{\xi_{j}} z_{\xi}|\cdot| v \mid d x \\
& +C \int_{\mathbf{R}^{n}}\left|z_{\xi}\right|^{p-2} \cdot\left|\partial_{\xi_{j}} z_{\xi}\right| \cdot|w| \cdot|v| d x
\end{aligned}
$$


Then, for $1<p \leq 2$,

$$
\begin{aligned}
& \int_{\mathbf{R}^{n}}|| z_{\xi}+\left.w\right|^{p-1}-\left|z_{\xi}\right|^{p-1}|\cdot| \partial_{\xi_{j}} z_{\xi}|\cdot| v \mid d x \\
\leq & C \int_{\mathbf{R}^{n}}|w|^{p-1} \cdot\left|\partial_{\xi_{j}} z_{\xi}\right| \cdot|v| d x \\
\leq & C\|w\|_{L^{p+1}}^{p-1}\left\|\partial_{\xi_{j}} z_{\xi}\right\|_{L^{p+1}}\|v\|_{L^{p+1}} \leq C\|w\|_{s}^{p-1}\|v\|_{s},
\end{aligned}
$$

and, for $2<p<\frac{n+2 s}{n-2 s}$ (if $2<\frac{n+2 s}{n-2 s}$ ),

$$
\begin{aligned}
& \int_{\mathbf{R}^{n}}|| z_{\xi}+\left.w\right|^{p-1}-\left|z_{\xi}\right|^{p-1}|\cdot| \partial_{\xi_{j}} z_{\xi}|\cdot| v \mid d x \\
\leq & \int_{\mathbf{R}^{n}}(p-1)\left|z_{\xi}+\theta_{3} w\right|^{p-2}|w| \cdot\left|\partial_{\xi_{j}} z_{\xi}\right| \cdot|v| d x \\
\leq & C\left\|z_{\xi}+\theta_{3} w\right\|_{L^{p+1}}^{p-2}\left\|\partial_{\xi_{j}} z_{\xi}\right\|_{L^{p+1}}\|w\|_{L^{p+1}}\|v\|_{L^{p+1}} \leq C\|w\|_{s}\|v\|_{s} .
\end{aligned}
$$

Here $\theta_{3} \in[0,1]$. Then we have that

$$
\int_{\mathbf{R}^{n}}|| z_{\xi}+\left.w\right|^{p-1}-\left|z_{\xi}\right|^{p-1}|\cdot| \partial_{\xi_{j}} z_{\xi}|\cdot| v \mid d x \leq C\|w\|_{s}^{\sigma}\|v\|_{s},
$$

where $\sigma=\min \{1, p-1\}$. Furthermore, we estimate

$$
\begin{aligned}
& \int_{\mathbf{R}^{n}}\left|z_{\xi}\right|^{p-2} \cdot\left|\partial_{\xi_{j}} z_{\xi}\right| \cdot|w| \cdot|v| d x \\
\leq & C \int_{\mathbf{R}^{n}}\left(\frac{1}{(1+|x-\xi|)^{n+2 s}}\right)^{p-1} \cdot|w| \cdot|v| d x \\
\leq & C\|w\|_{0}\|v\|_{0} \leq C\|w\|_{s}\|v\|_{s} .
\end{aligned}
$$

Therefore, we obtain

$$
\left|T_{4}\right| \leq C\|w\|_{s}^{\sigma}\|v\|_{s}
$$

where $\sigma=\min \{1, p-1\}$.

Summarizing the estimates for $T_{1}, T_{2}, T_{3}, T_{4}$, we get

$$
\left\|\hat{\mathcal{L}} \partial_{\xi_{j}} w\right\|_{s} \leq C\left(\varepsilon|\nabla V(\varepsilon \xi)|+\|w\|_{s}^{\sigma}\right) .
$$

Then by Remark 5.6, it holds that

$$
\left\|\hat{\mathcal{L}} \partial_{\xi_{j}} w\right\|_{s} \leq C\left(\varepsilon|\nabla V(\varepsilon \xi)|+O\left(\varepsilon^{2}\right)\right)^{\sigma} .
$$

Thus, we finally obtain

$$
\left\|\nabla_{\xi} w\right\|_{s} \leq C\left(\varepsilon|\nabla V(\varepsilon \xi)|+O\left(\varepsilon^{2}\right)\right)^{\sigma} .
$$

This completes the proof. 
5.2. Analysis of $\Phi_{\varepsilon}(\xi)$. In this subsection, we shall expand $\Phi_{\varepsilon}(\xi)$. By the definition, we have that

$$
\begin{aligned}
\Phi_{\varepsilon}(\xi)= & \frac{1}{2}\left\|z_{\xi}+w(\varepsilon, \xi)\right\|_{s}^{2}+\frac{1}{2} \int_{\mathbf{R}^{n}} V(\varepsilon x)\left(z_{\xi}+w(\varepsilon, \xi)\right)^{2} d x \\
& -\frac{1}{p+1} \int_{\mathbf{R}^{n}}\left|z_{\xi}+w(\varepsilon, \xi)\right|^{p+1} d x
\end{aligned}
$$

Since $(-\Delta)^{s} z_{\xi}+z_{\xi}+V(\varepsilon \xi) z_{\xi}=z_{\xi}^{p}$, it holds that

$$
\left\langle z_{\xi}, w\right\rangle_{s}=-V(\varepsilon \xi) \int_{\mathbf{R}^{n}} z_{\xi} w d x+\int_{\mathbf{R}^{n}} z_{\xi}^{p} w d x .
$$

Therefore, we can rewrite

$$
\begin{aligned}
\Phi_{\varepsilon}(\xi)= & \left(\frac{1}{2}-\frac{1}{p+1}\right) \int_{\mathbf{R}^{n}} z^{p+1} d x+\frac{1}{2} \int_{\mathbf{R}^{n}}(V(\varepsilon x)-V(\varepsilon \xi)) z^{2} d x \\
& +\int_{\mathbf{R}^{n}}(V(\varepsilon x)-V(\varepsilon \xi)) z w d x+\frac{1}{2} \int_{\mathbf{R}^{n}} V(\varepsilon x) w^{2} d x \\
& +\frac{1}{2}\|w\|_{s}^{2}-\frac{1}{p+1} \int_{\mathbf{R}^{n}}\left(|z+w|^{p+1}-z^{p+1}-(p+1) z^{p} w\right) d x .
\end{aligned}
$$

By the definition of $z(x)$ (see Subsection 2.3), $z(x)=b(\varepsilon \xi) U(a(\varepsilon \xi) x)$ where $a(\varepsilon \xi)=$ $(1+V(\varepsilon \xi))^{\frac{1}{2 s}}$ and $b(\varepsilon \xi)=(1+V(\varepsilon \xi))^{\frac{1}{p-1}}$. Then we have that

$$
\int_{\mathbf{R}^{n}} z^{p+1} d x=C_{0}(1+V(\varepsilon \xi))^{\theta}
$$

where $C_{0}=\int_{\mathbf{R}^{n}} U^{p+1} d x$ and $\theta=\frac{p+1}{p-1}-\frac{n}{2 s}$. Let $C_{1}=\left(\frac{1}{2}-\frac{1}{p+1}\right) C_{0}$. Then

$$
\Phi_{\varepsilon}(\xi)=C_{1}(1+V(\varepsilon \xi))^{\theta}+\Gamma_{\varepsilon}(\xi)+\Psi_{\varepsilon}(\xi),
$$

where

$$
\Gamma_{\varepsilon}(\xi)=\frac{1}{2} \int_{\mathbf{R}^{n}}[V(\varepsilon x)-V(\varepsilon \xi)] z^{2} d x+\int_{\mathbf{R}^{n}}[V(\varepsilon x)-V(\varepsilon \xi)] z w d x
$$

and

$$
\begin{aligned}
\Psi_{\varepsilon}(\xi)= & \frac{1}{2} \int_{\mathbf{R}^{n}} V(\varepsilon x) w^{2} d x+\frac{1}{2}\|w\|_{s}^{2} \\
& -\frac{1}{p+1} \int_{\mathbf{R}^{n}}\left[|z+w|^{p+1}-z^{p+1}-(p+1) z^{p} w\right] d x .
\end{aligned}
$$

LEMMA 5.8. We have the following estimate:

$$
\left|\nabla \Psi_{\varepsilon}(\xi)\right| \leq C\|w\|_{s}\left(\|w\|_{s}^{\sigma}+\left\|\nabla_{\xi} w\right\|_{s}\right) .
$$


Proof. A direct calculus yields, for $j=1,2, \cdots, n$,

$$
\begin{aligned}
\mid \partial_{\xi_{j}}\left(\frac{1}{2} \int_{\mathbf{R}^{n}} V(\varepsilon x) w^{2} d x+\right. & \left.\frac{1}{2}\|w\|_{s}^{2}\right)|=| \int_{\mathbf{R}^{n}} V(\varepsilon x) w \partial_{\xi_{j}} w d x+\left\langle w, \partial_{\xi_{j}} w\right\rangle_{s} \mid \\
& \leq C\left(\|w\|_{0}\left\|\partial_{\xi_{j}} w\right\|_{0}+\|w\|_{s}\left\|\partial_{\xi_{j}} w\right\|_{s}\right) \leq C\left(\|w\|_{s}\left\|\partial_{\xi_{j}} w\right\|_{s}\right) .
\end{aligned}
$$

Estimate

$$
\begin{aligned}
& \left|\partial_{\xi_{j}}\left(\frac{1}{p+1} \int_{\mathbf{R}^{n}}\left(|z+w|^{p+1}-z^{p+1}-(p+1) z^{p} w\right) d x\right)\right| \\
= & \left|\int_{\mathbf{R}^{n}}\left(|z+w|^{p}\left(\partial_{\xi_{j}} z+\partial_{\xi_{j}} w\right)-z^{p}\left(\partial_{\xi_{j}} z+\partial_{\xi_{j}} w\right)-p z^{p-1} w \partial_{\xi_{j}} z\right) d x\right| \\
= & \left|\int_{\mathbf{R}^{n}}\left(p\left|z+\theta_{4} w\right|^{p-1} w\left(\partial_{\xi_{j}} z+\partial_{\xi_{j}} w\right)-p z^{p-1} w \partial_{\xi_{j}} z\right) d x\right| \\
= & \left|\int_{\mathbf{R}^{n}}\left(p w\left(\left|z+\theta_{4} w\right|^{p-1}-z^{p-1}\right) \partial_{\xi_{j}} z+p w\left|z+\theta_{4} w\right|^{p-1} \partial_{\xi_{j}} w\right) d x\right| .
\end{aligned}
$$

Here $\theta_{4} \in[0,1]$. Then, for $1<p \leq 2$,

$$
\begin{aligned}
& \left|\int_{\mathbf{R}^{n}}\left(p w\left(\left|z+\theta_{4} w\right|^{p-1}-z^{p-1}\right) \partial_{\xi_{j}} z d x\right)\right| \\
\leq & \int_{\mathbf{R}^{n}}\left|p w^{p} \partial_{\xi_{j}} z\right| d x \leq C\left\|\partial_{\xi_{j}} z\right\|_{L^{p+1}}\|w\|_{L^{p+1}}^{p} \leq C\|w\|_{s}^{p},
\end{aligned}
$$

and, for $2<p<\frac{n+2 s}{n-2 s}$ (if $2<\frac{n+2 s}{n-2 s}$ ),

$$
\begin{aligned}
& \left|\int_{\mathbf{R}^{n}}\left(p w \mid\left(z+\left.\theta_{4} w\right|^{p-1}-z^{p-1}\right) \partial_{\xi_{j}} z d x\right)\right| \\
\leq & \int_{\mathbf{R}^{n}}\left|p(p-1) w^{2}\right| z+\left.\theta_{5} w\right|^{p-2} \partial_{\xi_{j}} z \mid d x \\
\leq & C\left\|\partial_{\xi_{j}} z\right\|_{L^{p+1}}\left\|z+\theta_{5} w\right\|_{L^{p+1}}^{p-2}\|w\|_{L^{p+1}}^{2} \\
\leq & C\left\|\partial_{\xi_{j}} z\right\|_{s}\left\|z+\theta_{5} w\right\|_{s}^{p-2}\|w\|_{s}^{2} \leq C\|w\|_{s}^{2} .
\end{aligned}
$$

Here $\theta_{5} \in[0,1]$. Therefore,

$$
\left|\partial_{\xi_{j}}\left(\frac{1}{p+1} \int_{\mathbf{R}^{n}}\left(|z+w|^{p+1}-z^{p+1}-(p+1) z^{p} w\right) d x\right)\right| \leq C\|w\|_{s}^{1+\sigma} .
$$

Moreover,

$$
\left|\int_{\mathbf{R}^{n}} p w\right| z+\left.\theta_{4} w\right|^{p-1} \partial_{\xi_{j}} w d x \mid \leq C\left\|z+\theta_{4} w\right\|_{L^{p+1}}^{p-1}\|w\|_{L^{p+1}}\left\|\partial_{\xi_{j}}\right\|_{L^{p+1}} \leq C\|w\|_{s}\left\|\partial_{\xi_{j}} w\right\|_{s} .
$$

Therefore, we have that

$$
\left|\nabla \Psi_{\varepsilon}(\xi)\right| \leq C\|w\|_{s}\left(\|w\|_{s}^{\sigma}+\left\|\nabla_{\xi} w\right\|_{s}\right) .
$$

This completes the proof. 
LEMMA 5.9. It holds

$$
\left|\nabla \Gamma_{\varepsilon}(\xi)\right| \leq C \varepsilon^{1+\sigma}
$$

Proof. Compute

$$
\begin{aligned}
& \int_{\mathbf{R}^{n}}(V(\varepsilon x)-V(\varepsilon \xi)) z^{2} d x \\
= & \varepsilon \int_{\mathbf{R}^{n}} \nabla V(\varepsilon \xi) \cdot(x-\xi) z^{2} d x \\
& +\varepsilon^{2} \int_{\mathbf{R}^{n}} D^{2} V\left(\varepsilon \xi+\theta_{6}(\varepsilon-\xi)\right)[x-\xi, x-\xi] z^{2} d x \\
= & \varepsilon \int_{\mathbf{R}^{n}} \nabla V(\varepsilon \xi) \cdot y z^{2}(y) d x \\
& +\varepsilon^{2} \int_{\mathbf{R}^{n}} D^{2} V\left(\varepsilon \xi+\theta_{6}(\varepsilon-\xi)\right)[x-\xi, x-\xi] z^{2} d x \\
= & \varepsilon^{2} \int_{\mathbf{R}^{n}} D^{2} V\left(\varepsilon \xi+\theta_{6}(\varepsilon-\xi)\right)[x-\xi, x-\xi] z^{2} d x
\end{aligned}
$$

where $\theta_{6} \in[0,1]$. Since $V \in C_{b}^{3}\left(\mathbf{R}^{n}\right)$, it holds that

$$
\begin{aligned}
& \left|\partial_{\xi_{j}}\left(\int_{\mathbf{R}^{n}}(V(\varepsilon x)-V(\varepsilon \xi)) z^{2} d x\right)\right| \\
= & \varepsilon^{2}\left|\partial_{\xi_{j}}\left(\int_{\mathbf{R}^{n}} D^{2} V\left(\varepsilon \xi+\theta_{6}(\varepsilon-\xi)\right)[x-\xi, x-\xi] z^{2} d x\right)\right| \leq C \varepsilon^{2} .
\end{aligned}
$$

Estimate

$$
\begin{aligned}
& \left|\partial_{\xi_{j}} \int_{\mathbf{R}^{n}}[V(\varepsilon x)-V(\varepsilon \xi)] z w d x\right| \\
\leq & \varepsilon|\nabla V(\varepsilon \xi)| \int_{\mathbf{R}^{n}}|z w| d x+\int_{\mathbf{R}^{n}}|V(\varepsilon x)-V(\varepsilon \xi)|\left|\partial_{\xi_{j}} z\right||w| d x \\
& +\int_{\mathbf{R}^{n}}|V(\varepsilon x)-V(\varepsilon \xi)||z|\left|\partial_{\xi_{j}} w\right| d x \\
\leq & \varepsilon|\nabla V(\varepsilon \xi)||| w\left\|_{0}+\left(\int_{\mathbf{R}^{n}}|V(\varepsilon x)-V(\varepsilon \xi)|^{2}\left|\partial_{\xi_{j}} z\right|^{2} d x\right)^{\frac{1}{2}}\right\| w \|_{0} \\
& +\left(\int_{\mathbf{R}^{n}}|V(\varepsilon x)-V(\varepsilon \xi)|^{2}|z|^{2} d x\right)^{\frac{1}{2}}\left\|\partial_{\xi_{j}} w\right\|_{0} .
\end{aligned}
$$

Thus by Lemma 3.2, Remark 5.6 and Lemma 5.7, we have that

$$
\left|\nabla\left(\int_{\mathbf{R}^{n}}(V(\varepsilon x)-V(\varepsilon \xi)) z w d x\right)\right| \leq C \varepsilon\left(\varepsilon+\|w\|_{s}+\|\nabla w\|_{s}\right) \leq C \varepsilon^{1+\sigma} .
$$

Therefore, from Estimates (5.9) (5.10), Equation (5.8) holds. 
Let $\alpha(\varepsilon, \xi)=\theta C_{1}(1+V(\varepsilon \xi))^{\theta-1}$, where $\theta=\frac{p+1}{p-1}-\frac{n}{2 s}$. Then summarizing all conclusion above, we get the following proposition.

Proposition 5.10. It holds

$$
\nabla \Phi_{\varepsilon}(\xi)=\alpha(\varepsilon \xi) \varepsilon \nabla V(\varepsilon \xi)+\varepsilon^{1+\sigma} \varpi_{\varepsilon}(\xi),
$$

where $\varpi_{\varepsilon}(\xi)$ is a bounded function and $\sigma=\min \{1, p-1\}$.

REMARK 5.11. Using similar argument, we can prove that

$$
\Phi_{\varepsilon}(\xi)=C(1+V(\varepsilon \xi))^{\theta}+\gamma_{\varepsilon}(\xi),
$$

where $C>0, \theta=\frac{p+1}{p-1}-\frac{n}{2 s}$ and $\left|\gamma_{\varepsilon}(\xi)\right| \leq C\left(\varepsilon|\nabla V(\varepsilon \xi)|+\varepsilon^{2}\right)$.

\section{Proof of the main theorem}

In this section, we shall prove the main theorem by a classical perturbation result.

6.1. A multiplicity result by perturbation. Let $M \subset \mathbf{R}^{n}$ be a non-empty set. We denote by $M_{\delta}$ its $\delta$-neighbourhood. The cup length $l(M)$ of $M$ is defined by

$$
l(M)=1+\sup \left\{k \in \mathbf{N} \mid \exists \alpha_{1}, \cdots, \alpha_{k} \in \check{H}^{*}(M) \backslash 1, \alpha_{1} \cup \cdots \cup \alpha_{k} \neq 0\right\} .
$$

If no such class exists, we set $l(M)=1$. Here $\check{H}^{*}(M)$ is the Alexander cohomology of $M$ with real coefficients and $\cup$ denotes the cup product.

Assume that $V$ has a smooth manifold of critical points of $M$. According to Bott [8], we say that $M$ is non-degenerate critical manifold for $V$ if every $x \in M$ is a critical point of $V$ and the nullity of all $x \in M$ equals to the dimension of $M$.

Now we recall a classical perturbation result. For more details, see Theorem 6.4 of Chapter II in $[\mathbf{1 1}]$.

THEOREM 6.1. Let $h \in C^{2}\left(\mathbf{R}^{n}\right)$ and $\Sigma \subset \mathbf{R}^{n}$ be a smooth compact non-degenerate critical manifold of $h$. Let $W$ be a neighbourhood of $\Sigma$ and let $g \in C^{1}\left(\mathbf{R}^{n}\right)$. Then, if $\|h-g\|_{C^{1}(\bar{W})}$ is sufficiently small, the function $g$ has at least $l(\Sigma)$ critical points in $W$.

6.2. Proof of Theorem 1.1. With the preliminary considerations of the sections above, we now prove Theorem 1.1 by the abstract perturbation theorem above.

Proof of Theorem 1.1. Fix $\bar{\rho}>0$ such that $M \subset B_{\bar{\rho}}$. Since $M$ is a non-degenerate smooth critical manifold of $V$, it is a non-degenerate critical manifold of $C_{1}(1+V)^{\theta}$ as well. To use Theorem 6.1, we define

$$
h(\xi)=C_{1}(1+V(\xi))^{\theta},
$$

and

$$
g(\xi)=\Phi_{\varepsilon}\left(\frac{\xi}{\varepsilon}\right)
$$

Set $\Sigma=M$. Fix a $\delta$-neighbourhood $M_{\delta}$ of $M$ such that $M_{\delta} \subset B_{\bar{\rho}}$ and the only critical points of $V$ in $M_{\delta}$ are those of $M$. Let $W=M_{\delta}$. From Proposition 5.10 and Remark 5.11, 
the function $\Phi_{\varepsilon}(\cdot / \varepsilon)$ is converges to $h(\cdot)$ in $C^{1}(\bar{W})$ as $\varepsilon \rightarrow 0$. Then Theorem 6.1 yields the existence of at least $l(M)$ critical points of $g$ for $\varepsilon$ sufficiently small.

Let $\xi_{k} \in M_{\delta}$ be any of those critical points. Then $\xi_{k} / \varepsilon$ is a critical point of $\Phi_{\varepsilon}$ and Proposition 5.5 implies that

$$
u_{\varepsilon, \xi_{k}}(x)=z_{\xi_{k}}\left(x-\frac{\xi_{k}}{\varepsilon}\right)+w\left(\varepsilon, \xi_{k}\right)
$$

is a critical point of $f_{\varepsilon}$ and hence a solution of Equation (1.4). Thus

$$
u_{\varepsilon, \xi_{k}}\left(\frac{x}{\varepsilon}\right) \simeq z_{\xi_{k}}\left(\frac{x-\xi_{k}}{\varepsilon}\right)
$$

is a solution of Equation (1.3).

Any $\xi_{k}$ converges to some point $\xi_{k}^{*} \in M_{\delta}$ as $\varepsilon \rightarrow 0$. By Proposition 5.10, we have that $\xi_{k}^{*}$ is a stationary point of $V$. Then the choice of $M_{\delta}$ implies that $\xi_{k}^{*} \in M$. That is, $u_{\varepsilon, \xi_{k}}(x / \varepsilon)$ concentrates near a point of $M$. This completes the proof.

\section{Acknowledgments}

We are grateful to the anonymous referees for useful comments and suggestions. This work was supported by National Natural Science Foundation of China (No. 11401521) and Zhejiang Provincial Natural Science Foundation of China (LQ13A010003).

\section{References}

[1] Robert A. Adams. Sobolev spaces. Pure and Applied Mathematics, Vol. 65. Academic Press [A subsidiary of Harcourt Brace Jovanovich, Publishers], New York-London, 1975.

[2] A. Ambrosetti and S. Badiale. Variational perturbative methods and bifurcation of bounds states from the essential spectrum. Proc. Royal Soc. Edinburgh, 128(A):1131-1161, 1998.

[3] A. Ambrosetti, S. Badiale, and S. Cingolani. Semiclassical states of nonlinear Schrödinger equations. Arch. Rational Mech. Anal., 140(3):285-300, 1997.

[4] A. Ambrosetti, A. Malchiodi, and S. Secchi. Multiplicity results for some nonlinear Schrödinger equations with potentials. Arch. Ration. Mech. Anal., 159(3):253-271, 2001.

[5] Antonio Ambrosetti, Andrea Malchiodi, and Wei-Ming Ni. Singularly perturbed elliptic equations with symmetry: existence of solutions concentrating on spheres. I. Comm. Math. Phys., 235(3):427466, 2003.

[6] Abbas Bahri and Yan Yan Li. On a min-max procedure for the existence of a positive solution for certain scalar field equations in $\mathbf{R}^{N}$. Rev. Mat. Iberoamericana, 6(1-2):1-15, 1990.

[7] Abbas Bahri and Pierre-Louis Lions. On the existence of a positive solution of semilinear elliptic equations in unbounded domains. Ann. Inst. H. Poincaré Anal. Non Linéaire, 14(3):365-413, 1997.

[8] R. Bott. Nondegenerate critical manifolds. Annals of Math., 60:248-267, 1957.

[9] Jaeyoung Byeon and Zhi-Qiang Wang. Standing waves with a critical frequency for nonlinear Schrödinger equations. Arch. Ration. Mech. Anal., 165(4):295-316, 2002.

[10] Luis Caffarelli and Luis Silvestre. An extension problem related to the fractional Laplacian. Comm. Partial Differential Equations, 32(7-9):1245-1260, 2007.

[11] Kung-Ching Chang. Infinite-dimensional Morse theory and multiple solution problems. Progress in Nonlinear Differential Equations and their Applications, 6. Birkhäuser Boston Inc., Boston, MA, 1993. 
[12] Guoyuan Chen and Youquan Zheng. Concentration phenomenon for fractional nonlinear Schrödinger equations. Comm. Pure Appl. Anal., 13(6):2359-2376, 2014.

[13] S. Cingolani and M. Lazzo. Multiple semiclassical standing waves for a class of nonlinear Schrödinger equations. Topol. Methods Nonlinear Anal., 10(3-4):1-13, 1997.

[14] Juan Dávila, Manuel del Pino, Serena Dipierro, and Enrico Valdinoci. Concentration phenomena for the nonlocal Schrödinger equation with Dirichlet datum. arXiv:1403.4435.

[15] Juan Dávila, Manuel del Pino, and Juncheng Wei. Concentrating standing waves for the fractional nonlinear Schrödinger equation. J. Differential Equations, 256(2):858-892, 2014.

[16] Manuel del Pino and Patricio L. Felmer. Local mountain passes for semilinear elliptic problems in unbounded domains. Calc. Var. Partial Differential Equations, 4(2):121-137, 1996.

[17] Manuel del Pino and Patricio L. Felmer. Semiclassical states of nonlinear Schrödinger equations: a variational reduction method. Math. Ann., 324:1-32, 2002.

[18] Manuel del Pino, Michal Kowalczyk, and Jun-Cheng Wei. Concentration on curves for nonlinear Schrödinger equations. Comm. Pure Appl. Math., 60(1):113-146, 2007.

[19] Eleonora Di Nezza, Giampiero Palatucci, and Enrico Valdinoci. Hitchhiker's guide to the fractional Sobolev spaces. Bull. Sci. Math., 136(5):521-573, 2012.

[20] M. Fall, F. Mahmoudi, and E. Valdinoci. Ground states and concentration phenomena for the fractional Schrödinger equation. arXiv:1411.0576.

[21] M. Fall and E. Valdinoci. Uniqueness and nondegeneracy of positive solutions of $(-\Delta)^{s} u+u=u^{p}$ in $\mathbb{R}^{n}$ when $s$ is close to 1 . arXiv: 1301.4868 .

[22] P. Felmer, A. Quaas, and J. Tan. Positive solutions of the nonlinear Schrödinger equation with the fractional Laplacian. Proc. Roy. Soc. Edinburgh Sect. A, 142(6):1237-1262, 2012.

[23] Andreas Floer and Alan Weinstein. Nonspreading wave packets for the cubic Schrödinger equation with a bounded potential. J. Funct. Anal., 69(3):397-408, 1986.

[24] Rupert Frank, Enno Lenzmann, and Luis Silvestre. Uniqueness of radial solutions for the fractional Laplacian. arXiv:1302.2652.

[25] Rupert L. Frank and Enno Lenzmann. Uniqueness of non-linear ground states for fractional Laplacians in $\mathbb{R}$. Acta Math., 210(2):261-318, 2013.

[26] Massimo Grossi. On the number of single-peak solutions of the nonlinear Schrödinger equation. Ann. Inst. H. Poincaré Anal. Non Linéaire, 19(3):261-280, 2002.

[27] Changfeng Gui. Existence of multi-bumps solutions for nonlinear Schrödinger equations via variational method, 1996.

[28] X. Kang and J. Wei. On interacting bumps of semiclassical states of nonlinear Schrödinger equations. Adv. Differential Equations, 5(7-9):899-928, 2000.

[29] Nick Laskin. Fractional Schrödinger equation. Phys. Rev. E (3), 66(5):056108, 7, 2002.

[30] YanYan Li. On a singularly perturbed elliptic equation. Adv. Differential Equations, 2(6):955-980, 1997.

[31] F. Mahmoudi, A. Malchiodi, and M. Montenegro. Solutions to the nonlinear Schrödinger equations carrying momentum along a curve. Comm. Pure Appl. Math., 62(9):1155-1264, 2009.

[32] Y.-G. Oh. Stability of semiclassical bound states of nonlinear Schrödinger equations with potentials. Comm. Math. Phys., 121(1):11-33, 1989.

[33] Y.-G. Oh. On positive multi-lump bound states of nonlinear Schrödinger equations under multiple well potential. Comm. Math. Phys., 131(2):223-253, 1990.

[34] P. Rabinowitz. On a class of nonlinear schrödinger equations. Z. Angew. Math. Phys., 43:270-291, 1992.

[35] M. A. Shubin. Pseudodifferential operators and spectral theory. Springer-Verlag, Berlin, second edition, 2001. Translated from the 1978 Russian original by Stig I. Andersson.

[36] X. F. Wang. On concentration of positive bound states of nonlinear Schrödinger equations. Comm. Math. Phys., 153(2):229-244, 1993. 
School of Mathematics and Statistics, Zhejiang University of Finance \& Economics, Hangzhou 310018, Zhejiang, P. R. China

E-mail address: gychen@zufe.edu.cn 